\title{
BRAUER'S GENERALIZED DECOMPOSITION NUMBERS AND UNIVERSAL DEFORMATION RINGS
}

\author{
FRAUKE M. BLEHER
}

\begin{abstract}
The versal deformation ring $R(G, V)$ of a mod $p$ representation $V$ of a profinite group $G$ encodes all isomorphism classes of lifts of $V$ to representations of $G$ over complete local commutative Noetherian rings. We introduce a new technique for determining $R(G, V)$ when $G$ is finite which involves Brauer's generalized decomposition numbers.
\end{abstract}

\section{INTRODUCTION}

Let $k$ be an algebraically closed field of positive characteristic $p$, let $W=W(k)$ be the ring of infinite Witt vectors over $k$, and let $G$ be a finite group. An important question in the representation theory of $G$ is whether a finitely generated $k G$ module $V$ can be lifted to $W$. For example, Green's lifting theorem shows that this is possible if there are no non-trivial 2-extensions of $V$ by itself. A natural generalization of this question is to consider the functor which sends each complete local commutative Noetherian ring $R$ with residue field $k$ to the set of isomorphism classes of lifts of $V$ over $R$. If this functor is represented by a ring $R(G, V)$, we say that $R(G, V)$ is the universal deformation ring of $V$. More generally, one can always associate to $V$ a versal deformation $\operatorname{ring} R(G, V)$, whose precise definition is recalled in Section 2. It was shown in 4, Prop. 2.1] that if the stable endomorphism ring End $_{k G}(V)$ is isomorphic to $k$, then the versal deformation $\operatorname{ring} R(G, V)$ is always universal.

Apart from the fact that universal deformation rings of representations of finite groups give more insight into the representation theory of these finite groups, there is another important motivation for studying these universal deformation rings. Namely, universal deformation rings for finite groups provide a good test case for various conjectures concerning the ring theoretic properties of universal deformation rings for profinite Galois groups. For example, Flach asked whether there could be universal deformation rings which are not complete intersections (see 20]). In [5.6] it was shown that the universal deformation ring of the non-trivial irreducible mod 2 representation of the symmetric group $S_{4}$ is not a complete intersection; in fact it is not even Cohen-Macaulay. This led to infinitely many examples of real quadratic fields $L$ such that the universal deformation ring of the inflation of this representation to the Galois group over $L$ of the maximal totally unramified extension of $L$ is not a complete intersection. In [7, examples of finite groups $G$ and

Received by the editors December 3, 2012 and, in revised form, January 27, 2013.

2010 Mathematics Subject Classification. Primary 20C20; Secondary 20C15, 16G10.

Key words and phrases. Universal deformation rings, Brauer's generalized decomposition numbers, tame blocks, dihedral defect groups, semidihedral defect groups, generalized quaternion defect groups.

The author was supported in part by NSA Grant H98230-11-1-0131. 
$\bmod p$ representations of $G$ for every odd prime number $p$ were constructed such that the universal deformation rings of these representations are not complete intersections. The main advantage of computing universal deformation rings for representations of finite groups is that one can use deep results from modular representation theory due to Brauer, Erdmann [27, Linckelmann [33, 34, Carlson-Thévenaz [18, 19, and others.

In this paper, we propose a new method of determining universal deformation rings, using Brauer's generalized decomposition numbers. Brauer generalized the usual decomposition numbers in [10] to be able to express the values of ordinary irreducible characters not only on $p$-regular elements but also on $p$-singular elements in terms of Brauer characters. We will show how to use these generalized decomposition numbers to determine the universal deformation rings $R(G, V)$ for certain $\bmod p$ representations $V$ of finite groups $G$ whose stable endomorphisms are all given by scalars. The $V$ we consider are those for which Brauer's generalized decomposition numbers carry the most information; these $V$ are called maximally ordinary below. For maximally ordinary $V$, the generalized decomposition numbers enable us to find a family of Galois orbits of ordinary irreducible characters of $G$ which can be used to construct lifts of $V$ to large local rings in characteristic 0 . This provides a powerful tool for computing universal deformation rings. The need for the use of Brauer's generalized decomposition numbers is related to how much fusion of conjugacy classes of $p$-power order elements occurs in $G$.

Suppose $V$ is a $k G$-module whose stable endomorphism ring is isomorphic to $k$. Then $V$ has a unique non-projective indecomposable summand (up to isomorphism). Since the universal deformation $\operatorname{ring} R(G, V)$ only depends on this indecomposable direct summand by [4, Cor. 2.7], we may assume that $V$ is indecomposable. Hence there exists a unique $p$-block $B$ to which $V$ belongs. The case when $B$ has finite representation type has been fully studied in [4]. Therefore, we concentrate in this paper on the case when $B$ has infinite tame representation type. Let $D$ be a defect group of $B$. We say $V$ is maximally ordinary if the Brauer character of $V$ is the restriction of an ordinary irreducible character $\chi$ such that for every $\sigma \in D$ of maximal $p$-power order, Brauer's generalized decomposition numbers corresponding to $\sigma$ and $\chi$ do not all lie in $\{0, \pm 1\}$ (see Definition 3.2). This condition ensures that Brauer's generalized decomposition numbers carry enough information for our method to be applied to $V$.

The following theorem summarizes our main results; more precise statements can be found in Section 6] and in particular in Corollary 6.2 and Theorem 6.6.

Theorem 1.1. Suppose $G$ is a finite group, $B$ is a block of $k G$ of infinite tame representation type, and $D$ is a defect group of $B$ of order $p^{n}$. There exists an indecomposable $k G$-module $V$ belonging to $B$ whose stable endomorphism ring is isomorphic to $k$ and which is maximally ordinary if and only if $B$ is not local and $n \geq 4$. Moreover, the isomorphism class of every such $V$ can be described explicitly. Suppose $V$ is such a maximally ordinary module. There exists a monic polynomial $q_{n}(t) \in W[t]$ of degree $p^{n-2}-1$ which depends on $D$ but not on $V$ and which can be given explicitly such that either

(i) $R(G, V) / p R(G, V) \cong k[[t]] /\left(t^{p^{n-2}-1}\right)$, in which case $R(G, V) \cong W[[t]] /\left(q_{n}(t)\right)$, or

(ii) $R(G, V) / p R(G, V) \cong k[[t]] /\left(t^{p^{n-2}}\right)$, in which case $R(G, V) \cong W[[t]] /\left(t q_{n}(t), p q_{n}(t)\right)$. 
In all cases, the ring $R(G, V)$ is isomorphic to a subquotient ring of $W D$, and it is a complete intersection if and only if we are in case $(i)$.

In particular, Theorem 1.1 gives a positive answer to [4, Question 1.1] for all $B$ and $V$ considered in the theorem. A precise description of the maximally ordinary modules $V$ belonging to non-local $B$ is given in Corollary 6.2. A formula for the polynomials $q_{n}(t)$ can be found in Definition 5.3 and Remark 5.4.

The use of Brauer's generalized decomposition numbers provides a correction to some arguments in [2, Sect. 3.4], [3, Sect. 5], and [8, Sect. 3.2]. Namely, in these papers a formula for the values of the ordinary irreducible characters on elements in $D$ of maximal $p$-power order was assumed, which is true for principal infinite tame blocks but cannot be verified for arbitrary infinite tame blocks because there may be more fusion of $D$-conjugacy classes in $G$ when the blocks are not principal (see Remark 5.2).

While we have focused in this paper on determining universal deformation rings using modular representation theory, it would be interesting to consider how the latter can be generalized using the former. For instance, Brauer's theory of generalized decomposition numbers arises from trying to express the values of the character of a representation $M$ defined over a complete discrete valuation ring $A$ of characteristic 0 in terms of Brauer characters associated to $\bmod p$ representations. The representation $M$ is a specialization of a versal lift $U$ of the reduction $\bar{M}$ of $M$ modulo the maximal ideal of $A$. A natural generalization of Brauer's theory would express the values of the character of $U$ inside the versal deformation ring $R$ associated to $U$ in terms of Brauer character values and suitable versal decomposition numbers in $R$. In this way, the theory of versal deformations provides a wider frame in which to view Brauer's work on generalized decomposition numbers.

The paper is organized as follows. In Section 2 we recall the basic definitions of deformations and deformation rings. In Section 3, we give a brief introduction to Brauer's generalized decomposition numbers, as introduced in [10,11. We also give the precise definition of maximally ordinary modules (see Definition 3.2). In Example 3.4, we show that there are no maximally ordinary modules belonging to local blocks of infinite tame representation type. In Sections 4, 6, we prove Theorem 1.1 for non-local blocks as follows. We first recall in Section 4 the description of the quivers and relations of the basic algebras of all non-local blocks $B$ of infinite tame representation type. We will also need a classification of all maximally ordinary $k G$ modules $V$ belonging to $B$. Such $V$ come with ordinary irreducible characters $\chi_{V}$ of $G$ such that the restriction of $\chi_{V}$ to the $p$-regular conjugacy classes is the Brauer character of $V$. We describe results of Brauer and Olsson in Section [5] which help identify all ordinary irreducible characters which can function as $\chi_{V}$. This leads then to a full description of all maximally ordinary modules $V$ in Corollary 6.2, For each such $V$, we need to work with all possible $\chi_{V}$ to construct a lift $U^{\prime}$ of $V$ over a large ring $R^{\prime}$ which we will eventually prove is close to being a universal lift of $V$. To prove the latter, we use Brauer's generalized decomposition numbers to show that a class sum associated to a well-chosen $p$-power order element provides an endomorphism of $U^{\prime}$ which nearly generates the universal deformation ring of $V$ as a $W$-algebra (see Lemma 5.6 and its use in Claim 4 in the proof of Theorem 6.6).

We would like to thank the referee for suggesting revisions which helped improve the paper. 


\section{Preliminaries}

In this section, we give a brief introduction to versal and universal deformation rings and deformations. For more background material, we refer the reader to 35 ] and 23 .

Let $k$ be an algebraically closed field of characteristic $p>0$, and let $W$ be the ring of infinite Witt vectors over $k$. Let $\hat{\mathcal{C}}$ be the category of all complete local commutative Noetherian rings with residue field $k$. Note that all rings in $\hat{\mathcal{C}}$ have a natural $W$-algebra structure. The morphisms in $\hat{\mathcal{C}}$ are continuous $W$-algebra homomorphisms which induce the identity map on $k$.

Suppose $G$ is a finite group and $V$ is a finitely generated $k G$-module. A lift of $V$ over an object $R$ in $\hat{\mathcal{C}}$ is a pair $(M, \phi)$, where $M$ is a finitely generated $R G$-module which is free over $R$ and $\phi: k \otimes_{R} M \rightarrow V$ is an isomorphism of $k G$-modules. Two lifts $(M, \phi)$ and $\left(M^{\prime}, \phi^{\prime}\right)$ of $V$ over $R$ are isomorphic if there is an isomorphism $f: M \rightarrow M^{\prime}$ with $\phi=\phi^{\prime} \circ(k \otimes f)$. The isomorphism class $[M, \phi]$ of a lift $(M, \phi)$ of $V$ over $R$ is called a deformation of $V$ over $R$, and the set of all such deformations is denoted by $\operatorname{Def}_{G}(V, R)$. The deformation functor

$$
\hat{F}_{V}: \hat{\mathcal{C}} \rightarrow \text { Sets }
$$

is a covariant functor which sends an object $R$ in $\hat{\mathcal{C}}$ to $\operatorname{Def}_{G}(V, R)$ and a morphism $\alpha: R \rightarrow R^{\prime}$ in $\hat{\mathcal{C}}$ to the map $\operatorname{Def}_{G}(V, R) \rightarrow \operatorname{Def}_{G}\left(V, R^{\prime}\right)$ defined by $[M, \phi] \mapsto$ $\left[R^{\prime} \otimes_{R, \alpha} M, \phi_{\alpha}\right]$, where $\phi_{\alpha}=\phi$ after identifying $k \otimes_{R^{\prime}}\left(R^{\prime} \otimes_{R, \alpha} M\right)$ with $k \otimes_{R} M$.

Suppose there exists an object $R(G, V)$ in $\hat{\mathcal{C}}$ and a deformation $\left[U(G, V), \phi_{U}\right]$ of $V$ over $R(G, V)$ with the following property: For each $R$ in $\hat{\mathcal{C}}$ and for each lift $(M, \phi)$ of $V$ over $R$ there exists a morphism $\alpha: R(G, V) \rightarrow R$ in $\hat{\mathcal{C}}$ such that $\hat{F}_{V}(\alpha)\left(\left[U(G, V), \phi_{U}\right]\right)=[M, \phi]$, and moreover $\alpha$ is unique if $R$ is the ring of dual numbers $k[\epsilon] /\left(\epsilon^{2}\right)$. Then $R(G, V)$ is called the versal deformation ring of $V$ and $\left[U(G, V), \phi_{U}\right]$ is called the versal deformation of $V$. If the morphism $\alpha$ is unique for all $R$ and all lifts $(M, \phi)$ of $V$ over $R$, then $R(G, V)$ is called the universal deformation ring of $V$ and $\left[U(G, V), \phi_{U}\right]$ is called the universal deformation of $V$. In other words, $R(G, V)$ is universal if and only if $R(G, V)$ represents the functor $\hat{F}_{V}$ in the sense that $\hat{F}_{V}$ is naturally isomorphic to the Hom functor $\operatorname{Hom}_{\hat{\mathcal{C}}}(R(G, V),-)$.

Note that the above definition of deformations can be weakened as follows. Given a lift $(M, \phi)$ of $V$ over a ring $R$ in $\hat{\mathcal{C}}$, define the corresponding weak deformation to be the isomorphism class of $M$ as an $R G$-module, without taking into account the specific isomorphism $\phi: k \otimes_{R} M \rightarrow V$. In general, a weak deformation of $V$ over $R$ identifies more lifts than a deformation of $V$ over $R$ that respects the isomorphism $\phi$ of a representative $(M, \phi)$. However, if the stable endomorphism ring $\operatorname{End}_{k G}(V)$ is isomorphic to $k$, these two definitions of deformations coincide (see [3, Remark 2.1]).

By [35, every finitely generated $k G$-module $V$ has a versal deformation ring. Since $G$ is a finite group, we have the following sufficient criterion for the universality of $R(G, V)$ :

Proposition 2.1 (4, Prop. 2.1]). Suppose $V$ is a finitely generated $k G$-module whose stable endomorphism ring $\underline{\operatorname{End}}_{k G}(V)$ is isomorphic to $k$. Then $V$ has a universal deformation ring $R(G, V)$. 


\section{BRAUER's GENERALIZED DECOMPOSITION NUMBERS}

In this section, we give a brief introduction to Brauer's generalized decomposition numbers, emphasizing the results needed in this paper. Throughout this section, let $p$ be a fixed prime number and let $G$ be a finite group such that $p$ divides $\# G$. Let $P$ be a fixed Sylow $p$-subgroup of $G$.

Brauer introduced generalized decomposition numbers in [10] to be able to express the values of the ordinary irreducible characters of $G$ on all conjugacy classes by means of the absolutely irreducible $p$-modular characters of certain subgroups of $G$. More precisely, let $g \in G$ and write $g$ (uniquely) as $g=u v$, where $u$ is a $p$-element of order $p^{\alpha}$ and $v$ is a $p$-regular element in the centralizer $C_{G}(u)$ (where we allow $u$ or $v$ to be identity elements). Let $\operatorname{IBr}\left(C_{G}(u)\right)$ denote the set of all absolutely irreducible $p$-modular characters of $C_{G}(u)$. By [10, Sect. 1], if $\chi$ is an ordinary irreducible character of $G$, then we have a formula

$$
\chi(u v)=\sum_{\varphi \in \operatorname{IBr}\left(C_{G}(u)\right)} d_{\chi, \varphi}^{(u)} \varphi(v),
$$

where the numbers $d_{\chi, \varphi}^{(u)}, \varphi \in \operatorname{IBr}\left(C_{G}(u)\right)$, are algebraic integers in the field of $p^{\alpha}$-th roots of unity which do not depend on $v$. These are called the generalized decomposition numbers of $G$ corresponding to $u$ and $\chi$.

If $u=1_{G}$, then the generalized decomposition numbers corresponding to $u$ are the usual decomposition numbers, which are non-negative integers, and (3.1) is the usual formula for the restriction to $p$-regular elements of an ordinary irreducible character $\chi$ in terms of an integral combination of the absolutely irreducible $p$ modular characters of $G=C_{G}\left(1_{G}\right)$.

We can use these generalized decomposition numbers to express the ordinary character table of $G$ as the product of two square matrices as follows. Recall that we fixed a Sylow $p$-subgroup $P$ of $G$. Let $u_{0}, u_{1}, \ldots, u_{h}$ be a complete system of representatives of $G$-conjugacy classes of $p$-elements in $G$ with $u_{0}=1_{G}$ and $u_{i} \in P$ for all $1 \leq i \leq h$. For each $0 \leq i \leq h$, let $v_{i, 1}, \ldots, v_{i, \ell_{i}}$ be a complete system of representatives of $C_{G}\left(u_{i}\right)$-conjugacy classes of $p$-regular elements in $C_{G}\left(u_{i}\right)$ with $v_{i, 1}=1_{G}$. Then $\left\{u_{i} v_{i, j} \mid 0 \leq i \leq h, 1 \leq j \leq \ell_{i}\right\}$ is a complete set of representatives of the conjugacy classes of $G$. Moreover, for each $0 \leq i \leq h$, there are precisely $\ell_{i}$ absolutely irreducible $p$-modular characters of $C_{G}\left(u_{i}\right)$, which we denote by $\varphi_{i, 1}, \ldots, \varphi_{i, \ell_{i}}$. Let $\left\{\chi_{1}, \ldots, \chi_{c}\right\}$ be the set of all ordinary irreducible characters of $G$. Then Brauer's above formula (3.1) can be written as

$$
\chi_{s}\left(u_{i} v_{i, j}\right)=\sum_{t=1}^{\ell_{i}} d_{s, t}^{i} \varphi_{i, t}\left(v_{i, j}\right)
$$

for all $1 \leq s \leq c, 0 \leq i \leq h, 1 \leq j \leq \ell_{i}$, where $d_{s, t}^{i}=d_{\chi_{s}, \varphi_{i, t}}^{\left(u_{i}\right)}$ for all $1 \leq t \leq \ell_{i}$. Write the conjugacy class representatives in the order

$$
u_{0} v_{0,1}, \ldots, u_{0} v_{0, \ell_{0}}, u_{1} v_{1,1}, \ldots, u_{1} v_{1, \ell_{1}}, \ldots, u_{h} v_{h, 1}, \ldots, u_{h} v_{h, \ell_{h}}
$$

Using (3.2), we obtain that the ordinary character table $\mathcal{X}$ can be written as a product $\mathcal{X}=\Delta \cdot \Phi$, where $\Delta$ contains the generalized decomposition numbers and 
$\Phi$ is a block diagonal matrix

$$
\Phi=\left(\begin{array}{ccc}
\Phi_{0} & & 0 \\
& \ddots & \\
0 & & \Phi_{h}
\end{array}\right)
$$

with

$$
\Phi_{i}=\left(\begin{array}{ccc}
\varphi_{i, 1}\left(v_{i, 1}\right) & \cdots & \varphi_{i, 1}\left(v_{i, \ell_{i}}\right) \\
\vdots & & \vdots \\
\varphi_{i, \ell_{i}}\left(v_{i, 1}\right) & \cdots & \varphi_{i, \ell_{i}}\left(v_{i, \ell_{i}}\right)
\end{array}\right)
$$

for all $0 \leq i \leq h$. By [11, Sect. 7, p. 45], the square of the determinant of $\Delta$ is $\pm p^{a}$ for some $a$, and the square of the determinant of $\Phi$ is an integer which is relatively prime to $p$.

Now fix a $p$-modular block $B$ of $G$ and suppose that there are $k(B)$ ordinary irreducible characters belonging to $B$. Reorder $\chi_{1}, \ldots, \chi_{c}$ such that $\chi_{1}, \ldots, \chi_{k(B)}$ belong to $B$. For each $0 \leq i \leq h$, reorder $\varphi_{i, 1} \ldots, \varphi_{i, \ell_{i}}$ such that the first $m_{i}$ characters, $\varphi_{i, 1}, \ldots, \varphi_{i, m_{i}}$, are precisely the absolutely irreducible $p$-modular characters belonging to blocks of $C_{G}\left(u_{i}\right)$ whose Brauer correspondents in $G$ are equal to $B$. (Note that for each block $b$ of $C_{G}\left(u_{i}\right)$, its Brauer correspondent $b^{G}$ in $G$ is well-defined since the centralizer in $G$ of a defect group of $b$ is contained in $C_{G}\left(u_{i}\right)$; see [1, Sect. 14].) It follows from [11, Sect. 6] that for $1 \leq s \leq k(B)$, (3.2) can be rewritten as

$$
\chi_{s}\left(u_{i} v_{i, j}\right)=\sum_{t=1}^{m_{i}} d_{s, t}^{i} \varphi_{i, t}\left(v_{i, j}\right)
$$

for all $0 \leq i \leq h, 1 \leq j \leq \ell_{i}$. In particular, we obtain for all $0 \leq i \leq h$ that

$$
\mathcal{X}_{B, i}=\left(\begin{array}{ccc}
\chi_{1}\left(u_{i} v_{i, 1}\right) & \cdots & \chi_{1}\left(u_{i} v_{i, \ell_{i}}\right) \\
\vdots & & \vdots \\
\chi_{k(B)}\left(u_{i} v_{i, 1}\right) & \cdots & \chi_{k(B)}\left(u_{i} v_{i, \ell_{i}}\right)
\end{array}\right)=\Delta_{B, i} \cdot \Phi_{i}
$$

where

$$
\Delta_{B, i}=\left(\begin{array}{cccccc}
d_{1,1}^{i} & \cdots & d_{1, m_{i}}^{i} & 0 & \cdots & 0 \\
\vdots & & \vdots & \vdots & & \vdots \\
d_{k(B), 1}^{i} & \cdots & d_{k(B), m_{i}}^{i} & 0 & \cdots & 0
\end{array}\right)
$$

and we assume, as above, that $\varphi_{i, 1}, \ldots, \varphi_{i, m_{i}}$ are precisely the absolutely irreducible $p$-modular characters belonging to blocks of $C_{G}\left(u_{i}\right)$ whose Brauer correspondents in $G$ are equal to $B$.

Now let $k$ be an algebraically closed field of characteristic $p$ and view $B$ as a block of $k G$. Let $W=W(k)$ be the ring of infinite Witt vectors over $k$ and let $F$ be the fraction field of $W$. In particular, $W$ contains all roots of unity of order not divisible by $p$. Let $\bar{F}$ be a fixed algebraic closure of $F$, and let $\xi$ be a root of unity in $\bar{F}$ whose order is a power of $p$ such that $F(\xi)$ is a splitting field for $G$. Then $W[\xi]$ is the ring of integers of $F(\xi)$, and we can view the ordinary character table $\mathcal{X}$ of $G$ and the matrix $\Delta$ of generalized decomposition numbers as taking values in $W[\xi]$, and the matrix $\Phi$ in (3.3) as taking values in $W$. Since the square of the determinant of $\Phi$ is an integer that is relatively prime to $p$, it follows that 
the determinant of $\Phi$, and hence the determinant of $\Phi_{i}$ for all $0 \leq i \leq h$, is a unit in $W$. Hence we can solve (3.5) for $\Delta_{B, i}$ to obtain

$$
\Delta_{B, i}=\mathcal{X}_{B, i} \cdot \Psi_{i}
$$

for all $0 \leq i \leq h$, where $\Psi_{i}=\Phi_{i}^{-1}$ is a matrix with values in $W$. In particular, (3.6) shows that if we reduce our discussion to a single block $B$, then we can replace $\xi$ by a $p$-power order root of unity $\zeta$ in $\bar{F}$ such that all ordinary irreducible characters of $G$ belonging to $B$ are realizable over $F(\zeta)$, i.e. they correspond to absolutely irreducible $F(\zeta) G$-modules.

Remark 3.1. Equations (3.1) and (3.4) can be rewritten to reflect the influence of fusion of $P$-conjugacy classes in $G$ (see [13, Sect. 1]). As before, let $u$ be a $p$-element of $G$ and let $v$ be a $p$-regular element in $C_{G}(u)$. Assuming the notation of the previous paragraph, let $\chi$ be an irreducible $F(\xi)$-character which belongs to a block $B$ of $k G$. Recall that a subsection $\left(y, b_{y}\right)$ for $B$ is a pair consisting of a p-element $y$ of $G$ and a block $b_{y}$ of $k C_{G}(y)$ with $b_{y}^{G}=B$. We obtain

$$
\chi(u v)=\sum_{\left(y, b_{y}\right)} \sum_{\varphi \in \operatorname{IBr}\left(b_{y}\right)} d_{\chi, \varphi}^{(y)} \varphi\left(z_{y} v z_{y}^{-1}\right),
$$

where $\left(y, b_{y}\right)$ ranges over a system of representatives for the conjugacy classes of subsections for $B$ such that $y$ is conjugate to $u$ in $G$, say $u=z_{y}^{-1} y z_{y}$. For each $\left(y, b_{y}\right), \varphi$ ranges over the absolutely irreducible $p$-modular characters associated with $b_{y}$.

Definition 3.2. Let $k$ be an algebraically closed field of characteristic $p$, let $W$ be the ring of infinite Witt vectors over $k$, and let $\bar{F}$ be a fixed algebraic closure of the fraction field $F$ of $W$. Suppose $B$ is a block of $k G$ and that $D$ is a defect group of $B$. Let $\zeta \in \bar{F}$ be a root of unity of $p$-power order such that all ordinary irreducible characters of $G$ belonging to $B$ are realizable over $F(\zeta)$. In particular, the ordinary (resp. $p$-modular) characters belonging to $B$ can be viewed as taking values in $W[\zeta]$ (resp. $W$ ). Suppose $V$ is an indecomposable $k G$-module whose stable endomorphism ring is isomorphic to $k$ and which belongs to $B$. We say $V$ is maximally ordinary if the $p$-modular character of $V$ is the restriction to the $p$ regular elements of an ordinary irreducible character $\chi$ such that for every $\sigma \in D$ of maximal $p$-power order there exists an absolutely irreducible $p$-modular character $\varphi$ of $C_{G}(\sigma)$ such that $d_{\chi, \varphi}^{(\sigma)} \notin\{0, \pm 1\}$.

Example 3.3. Let $k, W, F, B$ and $D$ be as in Definition 3.2, and suppose $B$ is a block of $k G$ of finite representation type, i.e. $D$ is cyclic. Assume $D=\langle\sigma\rangle$ has order $p^{a}$, where $a \geq 1$, and there are $e$ isomorphism classes of simple $B$-modules. Define $m=\left(p^{a}-1\right) / e$.

If $m=1$, it follows from [22, (1.7) and (1.8)] that $d_{\chi, \varphi}^{(\sigma)} \in\{0, \pm 1\}$ for all ordinary irreducible characters $\chi$ belonging to $B$ and all absolutely irreducible $p$-modular characters $\varphi$ of $C_{G}(\sigma)$. Now let $m>1$. It follows from [22, $\left.\S 1\right]$ that there are $m$ ordinary irreducible characters $\chi_{\lambda}$ belonging to $B$ which correspond to the exceptional vertex of the Brauer tree of $B$ with multiplicity $m$. Since $m>1$, it follows from [22, (1.7)] that for at least one of these $m$ ordinary irreducible characters $\chi_{\lambda}$, there exists an absolutely irreducible $p$-modular character $\varphi_{0}$ of $C_{G}(\sigma)$ such that 
$d_{\chi_{\lambda}, \varphi_{0}}^{(\sigma)} \notin\{0, \pm 1\}$. Moreover, by [22, (1.8)], if $\chi$ is a non-exceptional ordinary irreducible character belonging to $B$, then $d_{\chi, \varphi}^{(\sigma)} \in\{0, \pm 1\}$ for all absolutely irreducible $p$-modular characters $\varphi$ of $C_{G}(\sigma)$.

Let $V$ be an indecomposable $k G$-module whose stable endomorphism ring is isomorphic to $k$ and which belongs to $B$. It follows that $V$ is maximally ordinary if and only if $m>1$ and $V$ is a uniserial $B$-module having as composition factors the simple modules corresponding to the edges adjacent to the exceptional vertex of the Brauer tree of $B$, with each composition factor appearing exactly once. By [4, §3], all the maximally ordinary modules lie in the same $\Omega$-orbit, which is denoted by $E_{1}(B)$ in [4, Def. 3.1].

Suppose $m>1$. Using similar arguments as described in Sections [4 6] below, we can use the generalized decomposition numbers for cyclic blocks, as given in [22], to reprove some of the results in [4]. Namely, we can determine the universal deformation rings $R(G, V)$ for all maximally ordinary $k G$-modules $V$ belonging to the cyclic block $B$. If $e>1$, the $m$ exceptional ordinary irreducible characters $\chi_{\lambda}$ play the most important role. The main idea is to use the $\chi_{\lambda}$ to find a lift of $V$ over a $W$-algebra of the form $W[[t]] /\left(q_{a}(t)\right)$, where $q_{a}(t) \in W[t]$ is a monic polynomial of degree $m$ which is the product of the minimal polynomials over $F$ of certain generalized decomposition numbers corresponding to the $\chi_{\lambda}$. If $e=1$, one also has to take into account the single non-exceptional ordinary irreducible character belonging to $B$ to construct a lift of $V$ over $W D$. In both cases, one then uses the determination of $R(G, V) / p R(G, V)$ to establish that $W[[t]] /\left(q_{a}(t)\right)$ (resp. $W D$ ) is isomorphic to $R(G, V)$ when $e>1$ (resp. $e=1$ ).

Example 3.4. Let $k, W, F, B$ and $D$ be as in Definition 3.2, and suppose $B$ is a block of $k G$ of infinite tame representation type. Let $V$ be an indecomposable $k G$-module whose stable endomorphism ring is isomorphic to $k$ and which belongs to $B$.

If $B$ is not local, a precise description of all $V$ which are maximally ordinary will be given in Corollary 6.2 (see also Lemma 6.1).

Suppose now that $B$ is local, i.e. there is a unique absolutely irreducible $p$ modular character $\psi$ belonging to $B$, and suppose $D$ has order $p^{n}$. If $n=2$ (resp. $n=3$ ), it follows from [14, Sect. VII] (resp. [15, 36]) that all generalized decomposition numbers corresponding to maximal $p$-power order elements in $D$ lie in $\{0, \pm 1\}$. Hence there are no maximally ordinary $k G$-modules belonging to $B$ if $n \leq 3$. Now suppose $n \geq 4$. Let $\sigma \in D$ be of maximal $p$-power order, and let $\chi$ be an ordinary irreducible character belonging to $B$. It follows from [15, 36] that if $\chi$ has height 0 , then the non-zero generalized decomposition numbers corresponding to $\sigma$ and $\chi$ are \pm 1 . On the other hand, if $\chi$ does not have height 0 , then the restriction of $\chi$ to the $p$-regular conjugacy classes is equal to $2 \psi$. It follows that if $V$ is maximally ordinary, then it has to be uniserial of length 2. However, if $n \geq 4$, then the stable endomorphism ring of every uniserial $B$-module of length 2 has $k$-dimension at least 2 . Therefore, it follows that if $B$ is a local block of infinite tame representation type, then $V$ cannot be maximally ordinary.

\section{TAME BLOCKS}

By Example 3.4, there are no maximally ordinary modules (in the sense of Definition 3.2) belonging to local blocks of infinite tame representation type. Therefore, we make the following assumptions for the remainder of the paper. 
Hypothesis 4.1. Let $k$ be an algebraically closed field of positive characteristic $p$, let $W=W(k)$ be the ring of infinite Witt vectors over $k$, let $F$ be the fraction field of $F$, and let $\bar{F}$ be a fixed algebraic closure of $F$. Suppose $G$ is a finite group, $B$ is a non-local block of $k G$ of infinite tame representation type, and $D$ is a defect group of $B$ of order $p^{n}$. Let $\zeta$ be a primitive $p^{n-1}$-th root of unity in $\bar{F}$.

It follows from [9, 16, 29] that $p=2, n \geq 2$, and $D$ is dihedral, semidihedral or generalized quaternion. In particular, $n \geq 2$ if $D$ is dihedral, $n \geq 3$ if $D$ is generalized quaternion, and $n \geq 4$ if $D$ is semidihedral. Moreover, Brauer and Olsson proved that all ordinary irreducible characters of $G$ belonging to $B$ take values in $F(\zeta)$ (see [14, Prop. (7D)], [15, Prop. (5A)] and [36, Prop. 4.1]). In fact, all ordinary irreducible characters of $G$ belonging to $B$ can be realized by simple $F(\zeta) G$-modules (see Section [5).

Assume Hypothesis 4.1. By [15, 36, it follows that there are at most three isomorphism classes of simple $B$-modules. From Erdmann's classification of all blocks of tame representation type in [27, it follows that the quiver and relations of the basic algebra of $B$ can be given explicitly and that, up to Morita equivalence, there are 24 families of blocks $B$.

Using [24, 26, 27, 30, we now give a description of these families as follows. By [27, pp. 294-306], there are 12 possible quivers $Q$ which can occur for non-local basic algebras of dihedral, semidihedral or quaternion type: $2 \mathcal{A}, 2 \mathcal{B}, 3 \mathcal{A}, 3 \mathcal{B}, 3 \mathcal{C}, 3 \mathcal{D}$, $3 \mathcal{F}, 3 \mathcal{H}, 3 \mathcal{K}, 3 \mathcal{L}, 3 \mathcal{Q}, 3 \mathcal{R}$. For each such quiver $Q$, we combine Erdmann's results in [26, 27] and [30, Prop. 4.2] with Eisele's results in [24] to provide the most accurate description of a full set of representatives of basic algebras $\Lambda=k Q / I$ for the Morita equivalence classes of blocks $B$ as in Hypothesis 4.1 with Ext quiver $Q$.

We also provide the (usual) decomposition matrix for each block $B$. For better readability, all decomposition matrices appear at the end of the paper. As will be discussed in Section 5. $B$ always contains exactly 4 ordinary irreducible characters of height 0 and, unless $D$ is quaternion of order 8 , exactly $2^{n-2}-1$ ordinary irreducible characters of height 1 . If $D$ is quaternion of order $8, B$ contains exactly 3 ordinary irreducible characters of height 1 . If $n \geq 4$, then the family of $2^{n-2}-1$ ordinary irreducible characters of height 1 all define the same Brauer character on restricting to the 2-regular conjugacy classes of $G$. If $D$ is generalized quaternion or semidihedral, there may be additional ordinary irreducible characters of height $n-2$. In the decomposition matrices, we list first the 4 ordinary irreducible characters of height 0 , then the family of $2^{n-2}-1$ ordinary irreducible characters of height 1 , and then the ordinary irreducible characters of height $n-2$ if they exist. If $B$ has three isomorphism classes of simple modules, we moreover order the ordinary irreducible characters according to the sign conventions described in [15, Thm. 5] and [36. Thms. 4.10, 4.11 and 4.15].

To distinguish between different defect groups, we use the notation $\mathrm{D}(Q)$ (resp. $\mathrm{SD}(Q)$, resp. $\mathrm{Q}(Q))$ to mean that $\Lambda=k Q / I$ is Morita equivalent to a block $B$ with dihedral (resp. semidihedral, resp. generalized quaternion) defect groups.

\subsection{Blocks with quiver $2 \mathcal{A}$.}

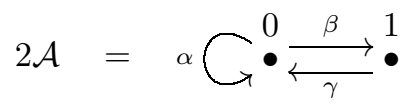


By [24 and [27, p. 294], if $B$ has dihedral defect groups and Ext quiver $2 \mathcal{A}$, then $B$ is Morita equivalent to $\mathrm{D}(2 \mathcal{A})=k[2 \mathcal{A}] / I_{\mathrm{D}(2 \mathcal{A})}$ where

$$
I_{\mathrm{D}(2 \mathcal{A})}=\left\langle\beta \gamma, \alpha^{2},(\gamma \beta \alpha)^{2^{n-2}}-(\alpha \gamma \beta)^{2^{n-2}}\right\rangle .
$$

The corresponding decomposition matrix is given in Figure 1 (Figures 1-18 are given in the Appendix).

By [27, p. 298], if $B$ has semidihedral defect groups and Ext quiver $2 \mathcal{A}$, then there exists $c \in k$ such that $B$ is Morita equivalent to either $\operatorname{SD}(2 \mathcal{A})_{1}(c)=$ $k[2 \mathcal{A}] / I_{\mathrm{SD}(2 \mathcal{A})_{1}, c}$ or $\mathrm{SD}(2 \mathcal{A})_{2}(c)=k[2 \mathcal{A}] / I_{\mathrm{SD}(2 \mathcal{A})_{2}, c}$, where

$$
\begin{aligned}
I_{\mathrm{SD}(2 \mathcal{A})_{1}, c}= & \left\langle\alpha^{2}-c(\gamma \beta \alpha)^{2^{n-2}}, \beta \gamma \beta-\beta \alpha(\gamma \beta \alpha)^{2^{n-2}-1}, \gamma \beta \gamma-\alpha \gamma(\beta \alpha \gamma)^{2^{n-2}-1},\right. \\
& \left.\alpha(\gamma \beta \alpha)^{2^{n-2}}\right\rangle, \\
I_{\mathrm{SD}(2 \mathcal{A})_{2}, c}= & \left\langle\beta \gamma, \alpha^{2}-\gamma \beta(\alpha \gamma \beta)^{2^{n-2}-1}-c(\gamma \beta \alpha)^{2^{n-2}},(\gamma \beta \alpha)^{2^{n-2}}-(\alpha \gamma \beta)^{2^{n-2}}\right\rangle .
\end{aligned}
$$

The decomposition matrix for $\operatorname{SD}(2 \mathcal{A})_{1}(c)$ is given in Figure 2 , and the decomposition matrix for $\operatorname{SD}(2 \mathcal{A})_{2}(c)$ is given in Figure 1 .

By [27, p. 303], if $B$ has generalized quaternion defect groups and Ext quiver $2 \mathcal{A}$, then there exists $c \in k$ such that $B$ is Morita equivalent to $\mathrm{Q}(2 \mathcal{A})(c)=$ $k[2 \mathcal{A}] / I_{\mathrm{Q}(2 \mathcal{A}), c}$, where

$$
\begin{aligned}
I_{\mathrm{Q}(2 \mathcal{A}), c}= & \left\langle\alpha^{2}-\gamma \beta(\alpha \gamma \beta)^{2^{n-2}-1}-c(\alpha \gamma \beta)^{2^{n-2}}, \beta \gamma \beta-\beta \alpha(\gamma \beta \alpha)^{2^{n-2}-1},\right. \\
& \left.\gamma \beta \gamma-\alpha \gamma(\beta \alpha \gamma)^{2^{n-2}-1}, \beta \alpha^{2}\right\rangle .
\end{aligned}
$$

The decomposition matrix for $\mathrm{Q}(2 \mathcal{A})(c)$ is given in Figure 2

\subsection{Blocks with quiver $2 \mathcal{B}$.}

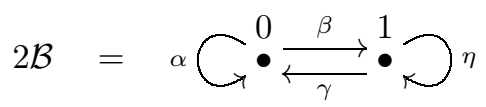

By [24 and [27, p. 295], if $B$ has dihedral defect groups and Ext quiver $2 \mathcal{B}$, then $B$ is Morita equivalent to $\mathrm{D}(2 \mathcal{B})=k[2 \mathcal{B}] / I_{\mathrm{D}(2 \mathcal{B})}$, where

$$
I_{\mathrm{D}(2 \mathcal{B})}=\left\langle\eta \beta, \gamma \eta, \beta \gamma, \alpha^{2}, \gamma \beta \alpha-\alpha \gamma \beta, \eta^{2^{n-2}}-\beta \alpha \gamma\right\rangle .
$$

The corresponding decomposition matrix is given in Figure 3 .

By [26, Lemmas (8.11) and (8.15)], 27, p. 299] and [30, Prop. 4.2], if $B$ has semidihedral defect groups and Ext quiver $2 \mathcal{B}$, then there exists $c \in k$ such that $B$ is Morita equivalent to either $\operatorname{SD}(2 \mathcal{B})_{1}(c)=k[2 \mathcal{B}] / I_{\mathrm{SD}(2 \mathcal{B})_{1}, c}$ or $\mathrm{SD}(2 \mathcal{B})_{2}(c)=$ $k[2 \mathcal{B}] / I_{\mathrm{SD}(2 \mathcal{B})_{2}, c}$ or $\mathrm{SD}(2 \mathcal{B})_{4}(c)=k[2 \mathcal{B}] / I_{\mathrm{SD}(2 \mathcal{B})_{4}, c}$, where

$$
\begin{aligned}
I_{\mathrm{SD}(2 \mathcal{B})_{1}, c}= & \left\langle\eta \beta, \gamma \eta, \beta \gamma, \alpha^{2}-\gamma \beta-c \alpha \gamma \beta, \gamma \beta \alpha-\alpha \gamma \beta, \eta^{2^{n-2}}-\beta \alpha \gamma\right\rangle, \\
I_{\mathrm{SD}(2 \mathcal{B})_{2}, c}= & \left\langle\eta \beta-\beta \alpha(\gamma \beta \alpha), \gamma \eta-\alpha \gamma(\beta \alpha \gamma), \alpha^{2}-c(\gamma \beta \alpha)^{2}, \beta \gamma-\eta^{2^{n-2}-1},\right. \\
& \left.\eta^{2} \beta, \gamma \eta^{2}\right\rangle, \\
I_{\mathrm{SD}(2 \mathcal{B})_{4}, c}=\langle & \gamma \eta-\alpha \gamma, \beta \alpha-\eta \beta, \alpha^{2^{n-2}+1}, \eta^{2^{n-2}+1}, \beta \alpha^{2^{n-2}-1}, \alpha^{2^{n-2}-1} \gamma, \\
& \left.\gamma \eta^{2^{n-2}-1}, \eta^{2^{n-2}-1} \beta, \gamma \beta-\alpha^{2}, \beta \gamma-\eta^{2}\left(1+c \eta^{2^{n-2}-2}\right)\right\rangle .
\end{aligned}
$$

The decomposition matrix for $\operatorname{SD}(2 \mathcal{B})_{1}(c)$ is given in Figure 3 , the decomposition matrix for $\operatorname{SD}(2 \mathcal{B})_{2}(c)$ is given in Figure 4 and the decomposition matrix for $\mathrm{SD}(2 \mathcal{B})_{4}(c)$ is given in Figure 5 .

By [27, IX.4.1 and pp. 303-304], if $B$ has generalized quaternion defect groups and Ext quiver $2 \mathcal{B}$, then there exists $c \in k$, respectively $p(t) \in k[t]$ with $p(0)=1$ 
and $a, c \in k$ with $a \neq 0$, such that $B$ is Morita equivalent to either $\mathrm{Q}(2 \mathcal{B})_{1}(c)=$ $k[2 \mathcal{B}] / I_{\mathrm{Q}(2 \mathcal{B})_{1}, c}$ or $\mathrm{Q}(2 \mathcal{B})_{2}(p, a, c)=k[2 \mathcal{B}] / I_{\mathrm{Q}(2 \mathcal{B})_{2}, p, a, c}$, where

$$
\begin{aligned}
I_{\mathrm{Q}(2 \mathcal{B})_{1}, c}=\langle & \eta \beta-\beta \alpha(\gamma \beta \alpha), \gamma \eta-\alpha \gamma(\beta \alpha \gamma), \alpha^{2}-\gamma \beta(\alpha \gamma \beta)-c(\alpha \gamma \beta)^{2}, \\
& \left.\beta \gamma-\eta^{2^{n-2}-1}, \beta \alpha^{2}\right\rangle, \\
I_{\mathrm{Q}(2 \mathcal{B})_{2}, p, a, c}=\langle & \gamma \eta-\alpha \gamma, \beta \alpha-\eta \beta, \alpha^{2^{n-2}+1}, \eta^{2^{n-2}+1}, \beta \alpha^{2^{n-2}-1}, \alpha^{2^{n-2}-1} \gamma, \\
& \left.\gamma \beta-p(\alpha) \alpha^{2}, \beta \gamma-p(\eta) \eta^{2}-a \eta^{2^{n-2}-1}-c \eta^{2^{n-2}}\right\rangle .
\end{aligned}
$$

The decomposition matrix for $\mathrm{Q}(2 \mathcal{B})_{1}(c)$ is given in Figure 4 and the decomposition matrix for $\mathrm{Q}(2 \mathcal{B})_{2}(p, a, c)$ is given in Figure 5. Note that by [36. Lemma 3.3], $\mathrm{Q}(2 \mathcal{B})_{2}(p, a, c)$ cannot actually occur as a block.

\subsection{Blocks with quiver $3 \mathcal{A}$.}

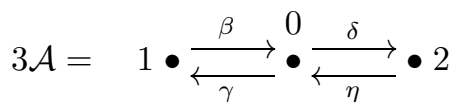

By 27, IX.5.4 and p. 295], if $B$ has dihedral defect groups and Ext quiver $3 \mathcal{A}$, then $B$ is Morita equivalent to $\mathrm{D}(3 \mathcal{A})_{1}=k[3 \mathcal{A}] / I_{\mathrm{D}(3 \mathcal{A})_{1}}$, where

$$
I_{\mathrm{D}(3 \mathcal{A})_{1}}=\left\langle\gamma \beta, \delta \eta,(\eta \delta \beta \gamma)^{2^{n-2}}-(\beta \gamma \eta \delta)^{2^{n-2}}\right\rangle .
$$

The corresponding decomposition matrix is given in Figure 6 .

By [27, IX.5.3 and pp. 299-300], if $B$ has semidihedral defect groups and Ext quiver $3 \mathcal{A}$, then $B$ is Morita equivalent to $\operatorname{SD}(3 \mathcal{A})_{1}=k[3 \mathcal{A}] / I_{\mathrm{SD}(3 \mathcal{A})_{1}}$, where

$$
I_{\mathrm{SD}(3 \mathcal{A})_{1}}=\left\langle\gamma \beta, \delta \eta \delta-\delta \beta \gamma(\eta \delta \beta \gamma)^{2^{n-2}-1}, \eta \delta \eta-\beta \gamma \eta(\delta \beta \gamma \eta)^{2^{n-2}-1}\right\rangle .
$$

The decomposition matrix for $\operatorname{SD}(3 \mathcal{A})_{1}$ is given in Figure 7 .

By [27, IX.5.2 and pp. 304-305], if $B$ has generalized quaternion defect groups and Ext quiver $3 \mathcal{A}$, then $B$ is Morita equivalent to $\mathrm{Q}(3 \mathcal{A})_{2}=k[3 \mathcal{A}] / I_{\mathrm{Q}(3 \mathcal{A})_{2}}$, where

$$
\begin{array}{r}
I_{\mathrm{Q}(3 \mathcal{A})_{2}=}\left\langle\beta \gamma \beta-\eta \delta \beta(\gamma \eta \delta \beta)^{2^{n-2}-1}, \gamma \beta \gamma-\gamma \eta \delta(\beta \gamma \eta \delta)^{2^{n-2}-1}, \delta \beta \gamma \beta,\right. \\
\left.\quad \delta \eta \delta-\delta \beta \gamma(\eta \delta \beta \gamma)^{2^{n-2}-1}, \eta \delta \eta-\beta \gamma \eta(\delta \beta \gamma \eta)^{2^{n-2}-1}, \gamma \eta \delta \eta\right\rangle .
\end{array}
$$

The decomposition matrix for $\mathrm{Q}(3 \mathcal{A})_{2}$ is given in Figure 8 ,

\subsection{Blocks with quiver $3 \mathcal{B}$.}

$$
3 \mathcal{B}=\alpha \hookrightarrow^{1} \stackrel{\beta}{\longleftarrow} \stackrel{0}{\longleftarrow} \bullet \frac{\delta}{\longleftarrow} \bullet 2
$$

By [27, IX.5.4 and pp. 295-296], if $B$ has dihedral defect groups and Ext quiver $3 \mathcal{B}$, then $B$ is Morita equivalent to $\mathrm{D}(3 \mathcal{B})_{1}=k[3 \mathcal{B}] / I_{\mathrm{D}(3 \mathcal{B})_{1}}$, where

$$
I_{\mathrm{D}(3 \mathcal{B})_{1}}=\left\langle\beta \alpha, \alpha \gamma, \gamma \beta, \delta \eta, \eta \delta \beta \gamma-\beta \gamma \eta \delta, \alpha^{2^{n-2}}-\gamma \eta \delta \beta\right\rangle .
$$

The corresponding decomposition matrix is given in Figure 9 .

By [27, p. 300], if $B$ has semidihedral defect groups and Ext quiver $3 \mathcal{B}$, then $B$ is Morita equivalent to either $\mathrm{SD}(3 \mathcal{B})_{1}=k[3 \mathcal{B}] / I_{\mathrm{SD}(3 \mathcal{B})_{1}}$ or $\mathrm{SD}(3 \mathcal{B})_{2}=k[3 \mathcal{B}] / I_{\mathrm{SD}(3 \mathcal{B})_{2}}$, where

$$
\begin{aligned}
& I_{\mathrm{SD}(3 \mathcal{B})_{1}}=\left\langle\beta \alpha, \alpha \gamma, \gamma \beta, \delta \eta \delta-\delta \beta \gamma, \eta \delta \eta-\beta \gamma \eta, \alpha^{2^{n-2}}-\gamma \eta \delta \beta\right\rangle, \\
& I_{\mathrm{SD}(3 \mathcal{B})_{2}}=\left\langle\delta \eta, \gamma \beta-\alpha^{2^{n-2}-1}, \alpha \gamma-\gamma \eta \delta(\beta \gamma \eta \delta), \beta \alpha-\eta \delta \beta(\gamma \eta \delta \beta)\right\rangle .
\end{aligned}
$$


The decomposition matrix for $\mathrm{SD}(3 \mathcal{B})_{1}$ is given in Figure 10, and the decomposition matrix for $\mathrm{SD}(3 \mathcal{B})_{2}$ is given in Figure 11

By [27, p. 305], if $B$ has generalized quaternion defect groups and Ext quiver $3 \mathcal{B}$, then $B$ is Morita equivalent to $\mathrm{Q}(3 \mathcal{B})=k[3 \mathcal{B}] / I_{\mathrm{Q}(3 \mathcal{B})}$, where

$$
\begin{aligned}
I_{\mathrm{Q}(3 \mathcal{B})}= & \left\langle\gamma \beta-\alpha^{2^{n-2}-1}, \alpha \gamma-\gamma \eta \delta(\beta \gamma \eta \delta), \beta \alpha-\eta \delta \beta(\gamma \eta \delta \beta), \delta \eta \delta-\delta \beta \gamma(\eta \delta \beta \gamma),\right. \\
& \left.\eta \delta \eta-\beta \gamma \eta(\delta \beta \gamma \eta), \beta \alpha^{2}, \delta \eta \delta \beta\right\rangle .
\end{aligned}
$$

The decomposition matrix for $\mathrm{Q}(3 \mathcal{B})$ is given in Figure 12 .

\subsection{Blocks with quiver $3 \mathcal{C}$.}

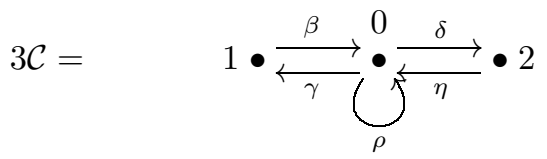

By [27, VI.5] (resp. by [27, IX.5.3 and p. 305]), there are no blocks $B$ with dihedral (resp. generalized quaternion) defect groups that have Ext quiver $3 \mathcal{C}$.

By [27, IX.5.3 and pp. 300-301], if $B$ has semidihedral defect groups and Ext quiver $3 \mathcal{C}$, then $B$ is Morita equivalent to either $\operatorname{SD}(3 \mathcal{C})_{2,1}=k[3 \mathcal{C}] / I_{\mathrm{SD}(3 \mathcal{C})_{2,1}}$ or $\mathrm{SD}(3 \mathcal{C})_{2,2}=k[3 \mathcal{C}] / I_{\mathrm{SD}(3 \mathcal{C})_{2,2}}$, where

$$
\begin{aligned}
& I_{\mathrm{SD}(3 \mathcal{C})_{2,1}}=\left\langle\rho \beta, \delta \rho, \rho \eta, \gamma \rho, \beta \gamma-\eta \delta,(\beta \gamma)^{2}-\rho^{2^{n-2}}, \delta \beta \gamma \beta, \gamma \eta \delta \eta\right\rangle, \\
& I_{\mathrm{SD}(3 \mathcal{C})_{2,2}}=\left\langle\rho \beta, \delta \rho, \rho \eta, \gamma \rho, \beta \gamma-\eta \delta,(\beta \gamma)^{2^{n-2}}-\rho^{2}, \delta \beta(\gamma \beta)^{2^{n-2}-1}\right. \text {, } \\
& \left.\gamma \eta(\delta \eta)^{2^{n-2}-1}\right\rangle \text {. }
\end{aligned}
$$

The decomposition matrix for $\mathrm{SD}(3 \mathcal{C})_{2,1}$ is given in Figure 13, and the decomposition matrix for $\mathrm{SD}(3 \mathcal{C})_{2,2}$ is given in Figure 14 .

\subsection{Blocks with quiver $3 \mathcal{D}$.}

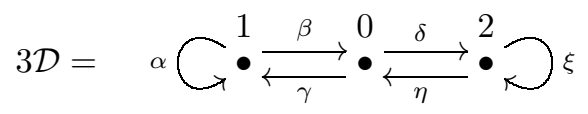

By [27, IX.5.1, IX.5.4 and p. 296] (resp. by [27, IX.5.1 and p. 306]), there are no blocks $B$ with dihedral (resp. generalized quaternion) defect groups that have Ext quiver $3 \mathcal{D}$.

By [27, p. 301], if $B$ has semidihedral defect groups and Ext quiver 3D, then $B$ is Morita equivalent to $\mathrm{SD}(3 \mathcal{D})=k[3 \mathcal{D}] / I_{\mathrm{SD}(3 \mathcal{D})}$, where

$$
I_{\mathrm{SD}(3 \mathcal{D})}=\left\langle\xi \delta, \eta \xi, \delta \eta, \gamma \beta-\alpha^{2^{n-2}-1}, \alpha \gamma-\gamma \eta \delta, \beta \alpha-\eta \delta \beta, \xi^{2}-\delta \beta \gamma \eta\right\rangle .
$$

The decomposition matrix for $\mathrm{SD}(3 \mathcal{D})$ is given in Figure 10.

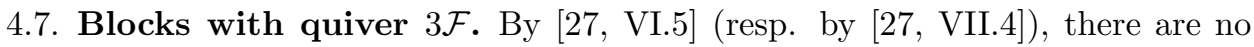
blocks $B$ with dihedral (resp. generalized quaternion) defect groups that have Ext quiver $3 \mathcal{F}$. By [27, IX.5.2 and p. 301], there are also no blocks $B$ with semidihedral defect groups that have Ext quiver $3 \mathcal{F}$. 


\subsection{Blocks with quiver $3 \mathcal{H}$.}

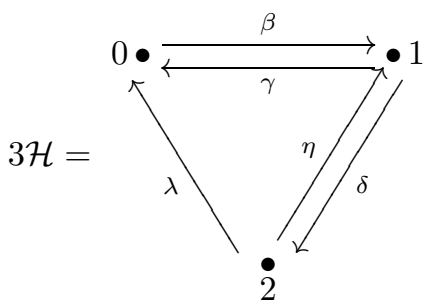

By [27, VI.5] (resp. by [27, VII.4]), there are no blocks $B$ with dihedral (resp. generalized quaternion) defect groups that have Ext quiver $3 \mathcal{H}$.

By [27, p. 301], if $B$ has semidihedral defect groups and Ext quiver $3 \mathcal{H}$, then $B$ is Morita equivalent to $\mathrm{SD}(3 \mathcal{H})_{1}=k[3 \mathcal{H}] / I_{\mathrm{SD}(3 \mathcal{H})_{1}}$ or $\mathrm{SD}(3 \mathcal{H})_{2}=k[3 \mathcal{H}] / I_{\mathrm{SD}(3 \mathcal{H})_{2}}$, where

$$
\begin{aligned}
& I_{\mathrm{SD}(3 \mathcal{H})_{1}}=\left\langle\lambda \delta-\gamma \beta \gamma, \beta \lambda-\eta(\delta \eta)^{2^{n-2}-1}, \eta \delta \beta, \delta \beta \gamma, \gamma \eta\right\rangle, \\
& I_{\mathrm{SD}(3 \mathcal{H})_{2}}=\left\langle\lambda \delta-\gamma(\beta \gamma)^{2^{n-2}-1}, \beta \lambda-\eta \delta \eta, \eta \delta \beta, \delta \beta \gamma, \gamma \eta\right\rangle .
\end{aligned}
$$

The decomposition matrix for $\mathrm{SD}(3 \mathcal{H})_{1}$ is given in Figure 15, and the decomposition matrix for $\mathrm{SD}(3 \mathcal{H})_{2}$ is given in Figure 16.

\subsection{Blocks with quiver $3 \mathcal{K}$.}

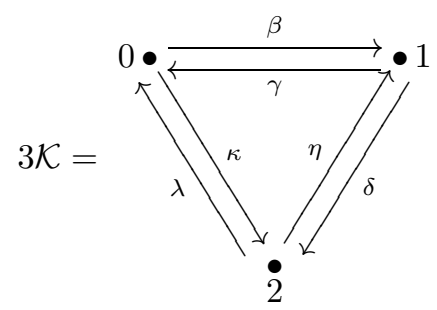

By [27, IX.5.2 and p. 302], there are no blocks $B$ with semidihedral defect groups that have Ext quiver $3 \mathcal{K}$.

By [27, p. 296], if $B$ has dihedral defect groups and Ext quiver $3 \mathcal{K}$, then $B$ is Morita equivalent to $\mathrm{D}(3 \mathcal{K})=k[3 \mathcal{K}] / I_{\mathrm{D}(3 \mathcal{K})}$, where

$$
I_{\mathrm{D}(3 \mathcal{K})}=\left\langle\delta \beta, \lambda \delta, \beta \lambda, \kappa \gamma, \eta \kappa, \gamma \eta, \gamma \beta-\lambda \kappa, \kappa \lambda-(\delta \eta)^{2^{n-2}},(\eta \delta)^{2^{n-2}}-\beta \gamma\right\rangle .
$$

The corresponding decomposition matrix is given in Figure 17.

By [27, p. 306], if $B$ has generalized quaternion defect groups and Ext quiver $3 \mathcal{K}$, then $B$ is Morita equivalent to $\mathrm{Q}(3 \mathcal{K})=k[3 \mathcal{K}] / I_{\mathrm{Q}(3 \mathcal{K})}$, where

$$
\begin{aligned}
I_{\mathrm{Q}(3 \mathcal{K})}= & \left\langle\delta \beta-\kappa \lambda \kappa, \gamma \eta-\lambda \kappa \lambda, \lambda \delta-\gamma \beta \gamma, \eta \kappa-\beta \gamma \beta, \beta \lambda-\eta(\delta \eta)^{2^{n-2}-1},\right. \\
& \left.\kappa \gamma-\delta(\eta \delta)^{2^{n-2}-1}, \delta \beta \gamma, \gamma \eta \delta, \eta \kappa \lambda\right\rangle .
\end{aligned}
$$

The decomposition matrix for $\mathrm{Q}(3 \mathcal{K})$ is given in Figure 18 ,

4.10. Blocks with quivers $3 \mathcal{L}, 3 \mathcal{Q}$ or $3 \mathcal{R}$. By [27, VIII.2.0] (resp. by [27, VII.4]), there are no blocks $B$ with semidihedral (resp. generalized quaternion) defect groups that have Ext quivers $3 \mathcal{L}, 3 \mathcal{Q}$ or $3 \mathcal{R}$. By [27, IX.5.4], there are also no blocks $B$ with dihedral defect groups that have Ext quivers $3 \mathcal{L}, 3 \mathcal{Q}$ or $3 \mathcal{R}$. 


\section{ORdinARY CHARACTERS BELONGING TO TAME BLOCKS}

Assume Hypothesis 4.1. In particular, $p=2, n \geq 2$, and $D$ is dihedral, semidihedral or generalized quaternion.

For $2 \leq \ell \leq n-1$, define $\zeta_{\ell}=\zeta^{2^{n-1-\ell}}$, so that $\zeta_{\ell}$ is a primitive $2^{\ell}$-th root of unity. It follows from [14, Sect. VII], 15, 36] and 28, (see below) that every ordinary irreducible character of $G$ which belongs to $B$ is realizable over $F(\zeta)$, i.e. it corresponds to an absolutely irreducible $F(\zeta) G$-module. In particular, if we are only interested in the ordinary irreducible characters of $G$ belonging to $B$, we can replace $\xi$ in the paragraph before Remark 3.1 by $\zeta$.

In [15] (resp. [36]), the ordinary irreducible characters of $G$ belonging to $B$ were analyzed if $n \geq 3$ and $D$ is dihedral (resp. semidihedral or generalized quaternion). In the notation of [15, Sect. 4] (resp. [36, Sect. 2]), this means that we are either in Case $(a a)$, or in Case $(a b)$ or $(b a)$; see [15, Thm. 2] (resp. [36, Thms. 3.14-3.17]). In particular, in Case $(a a)$ (resp. Case $(a b)$ or $(b a)$ ) there are precisely three (resp. two) isomorphism classes of simple $B$-modules. Note that Case $(b a)$ can only occur when $D$ is semidihedral.

Remark 5.1. (a) If $D$ is dihedral of order 4 , i.e. $n=2$, it follows from 25 ] that $B$ is Morita equivalent to either $\mathrm{D}(3 \mathcal{A})_{1}$ or $\mathrm{D}(3 \mathcal{K})$. Moreover, there are precisely 4 ordinary irreducible characters belonging to $B$ and they all have height 0 . In the decomposition matrices in Figures 6] and [17, these characters are $\chi_{1}, \chi_{2}, \chi_{3}, \chi_{4}$. By [14, Prop. (7D)] and [28], it follows that all these characters are realizable over $F$, i.e. they correspond to absolutely irreducible $F G$-modules.

(b) If $D$ is quaternion of order 8, i.e. $n=3$, it follows from [36, p. 220 and Thm. 3.17] that we are in Case $(a a)$ and that there are precisely 4 (resp. 3) ordinary irreducible characters of height 0 (resp. $1=n-2$ ) belonging to $B$. Moreover, $B$ is Morita equivalent to one of the algebras in $\left\{\mathrm{Q}(3 \mathcal{A})_{2}, \mathrm{Q}(3 \mathcal{K})\right\}$, and in the decomposition matrices in Figures 8 and 18 , the ordinary irreducible characters of height 0 (resp. 1) are

$$
\chi_{1}, \chi_{2}, \chi_{3}, \chi_{4} \quad\left(\text { resp. } \chi_{5,1}, \chi_{6}, \chi_{7}\right) \text {. }
$$

By [36, Prop. 4.2] and [28], all these characters are realizable over $F$, i.e. they correspond to absolutely irreducible $F G$-modules.

(c) If $D$ is not quaternion of order 8 , then there are precisely 4 (resp. $2^{n-2}-1$ ) ordinary irreducible characters of height 0 (resp. 1) belonging to $B$. If $D$ is dihedral, these are all ordinary irreducible characters belonging to $B$. If $D$ is semidihedral of order $\geq 16$, there are 0 (resp. 1) additional ordinary irreducible characters of height $n-2$ belonging to $B$ if we are in Case $(a b)$ (resp. Cases $(b a)$ or $(a a)$ ). If $D$ is generalized quaternion of order $\geq 16$, there are 1 (resp. 2) additional ordinary irreducible characters of height $n-2$ belonging to $B$ if we are in Case $(a b)$ (resp. Case $(a a)$ ). In the decomposition matrices in Figures 118, the ordinary irreducible characters of height 0 (resp. 1) are

$$
\chi_{1}, \chi_{2}, \chi_{3}, \chi_{4} \quad\left(\text { resp. } \chi_{5, i} \text { for } 1 \leq i \leq 2^{n-2}-1\right),
$$

whereas the ordinary irreducible characters of height $n-2$ are $\chi_{6}$ or $\chi_{6}, \chi_{7}$, provided they exist. 
Let $n \geq 3$, and let $\sigma$ be an element of order $2^{n-1}$ in $D$. By [15, 36, there exists a block $b_{\sigma}$ of $k C_{G}(\sigma)$ with $b_{\sigma}^{G}=B$ which contains a unique irreducible Brauer character $\varphi^{(\sigma)}$ such that the following is true. There is an ordering of $\left(1,2, \ldots, 2^{n-2}-1\right)$ such that for $1 \leq i \leq 2^{n-2}-1$, the generalized decomposition number of $G$ corresponding to $\sigma, \chi_{5, i}$ and $\varphi^{(\sigma)}$ has the form

$$
d_{\chi_{5, i}, \varphi^{(\sigma)}}^{(\sigma)}= \begin{cases}\zeta^{i}+\zeta^{-i} & \text { if } D \text { is dihedral or generalized quaternion, } \\ & \text { or if } D \text { is semidihedral and } i \text { is even, } \\ \zeta^{i}-\zeta^{-i} & \text { if } D \text { is semidihedral and } i \text { is odd with } i \leq 2^{n-3}-1 \\ \zeta^{-i}-\zeta^{i} & \text { if } D \text { is semidihedral and } i \text { is odd with } i \geq 2^{n-3}+1\end{cases}
$$

Note that the formulas in (5.8) for $D$ semidihedral and $i$ odd follow since the $D$ conjugacy classes of elements of order $2^{n-1}$ in $D$ are represented by

$$
\sigma^{1}, \sigma^{3}, \ldots, \sigma^{2^{n-3}-1}, \quad \sigma^{-\left(2^{n-3}+1\right)}, \sigma^{-\left(2^{n-3}+3\right)}, \ldots, \sigma^{-\left(2^{n-2}-1\right)} .
$$

If $D$ is quaternion of order 8 , we have that $d_{\chi, \varphi^{(\sigma)}}^{(\sigma)}=\zeta+\zeta^{-1}=0$ for $\chi \in$ $\left\{\chi_{5,1}, \chi_{6}, \chi_{7}\right\}$.

For $2 \leq \ell \leq n-2$ define $\nu_{\ell}=\zeta_{\ell}+\zeta_{\ell}^{-1}$, and define

$$
\nu_{n-1}= \begin{cases}\zeta+\zeta^{-1} & \text { if } D \text { is dihedral or generalized quaternion, } \\ \zeta-\zeta^{-1} & \text { if } D \text { is semidihedral. }\end{cases}
$$

Note that $W$ contains all roots of unity of order not divisible by 2 . Hence by [15, 36] and by 28, the ordinary irreducible characters of height 0 or $n-2$ belonging to $B$ correspond to simple $F G$-modules. On the other hand, the characters $\chi_{5, i}$, $i=1, \ldots, 2^{n-2}-1$, fall into $n-2$ Galois orbits $\mathcal{O}_{2}, \ldots, \mathcal{O}_{n-1}$ under the action of $\operatorname{Gal}\left(F\left(\nu_{n-1}\right) / F\right)$. Namely for $2 \leq \ell \leq n-1$,

$$
\mathcal{O}_{\ell}=\left\{\chi_{5,2^{n-1-\ell}(2 u-1)} \mid 1 \leq u \leq 2^{\ell-2}\right\} .
$$

The field generated by the character values of each $\xi_{\ell} \in \mathcal{O}_{\ell}$ over $F$ is $F\left(\nu_{\ell}\right)$. Hence by 28 , each $\xi_{\ell}$ corresponds to an absolutely irreducible $F\left(\nu_{\ell}\right) G$-module $X_{\ell}$. By [31, Satz V.14.9], this implies that for $2 \leq \ell \leq n-1$, the Schur index of each $\xi_{\ell} \in \mathcal{O}_{\ell}$ over $F$ is 1 . Hence we obtain $n-2$ non-isomorphic simple $F G$-modules $V_{2}, \ldots, V_{n-1}$ with characters $\rho_{2}, \ldots, \rho_{n-1}$ satisfying

$$
\rho_{\ell}=\sum_{\xi_{\ell} \in \mathcal{O}_{\ell}} \xi_{\ell}=\sum_{u=1}^{2^{\ell-2}} \chi_{5,2^{n-1-\ell}(2 u-1)} \quad \text { for } 2 \leq \ell \leq n-1 .
$$

By [31, Hilfssatz V.14.7], $\operatorname{End}_{F G}\left(V_{\ell}\right)$ is a commutative $F$-algebra isomorphic to the field generated over $F$ by the character values of any $\xi_{\ell} \in \mathcal{O}_{\ell}$. This means

$$
\operatorname{End}_{F G}\left(V_{\ell}\right) \cong F\left(\nu_{\ell}\right) \quad \text { for } 2 \leq \ell \leq n-1 .
$$

Suppose $v_{1}, \ldots, v_{l(\sigma)}$ form a complete system of representatives of $C_{G}(\sigma)$-conjugacy classes of 2-regular elements in $C_{G}(\sigma)$ with $v_{1}=1_{G}$. By (3.6), for all $1 \leq i \leq 2^{n-2}-1$, the generalized decomposition number of $G$ corresponding to $\chi_{5, i}$, $\sigma$ and $\varphi^{(\sigma)}$ can be written as a $W$-linear combination of $\chi_{5, i}\left(\sigma v_{1}\right), \ldots, \chi_{5, i}\left(\sigma v_{l(\sigma)}\right)$, say

$$
d_{\chi_{5, i}, \varphi^{(\sigma)}}^{(\sigma)}=\tilde{w}_{1} \cdot \chi_{5, i}\left(\sigma v_{1}\right)+\cdots+\tilde{w}_{l(\sigma)} \cdot \chi_{5, i}\left(\sigma v_{l(\sigma)}\right)
$$


for certain $\tilde{w}_{1}, \ldots, \tilde{w}_{l(\sigma)} \in W$ which are independent of $i \in\left\{1, \ldots, 2^{n-2}-1\right\}$.

By [15. Thm. 5] and [36. Prop. 4.6], the characters $\chi_{5, i}$ have the same degree $x$ and they are all of height 1 for $1 \leq i \leq 2^{n-2}-1$. Hence $x=2^{a-n+1} x^{*}$, where $\# G=2^{a} \cdot g^{*}$ and $x^{*}$ and $g^{*}$ are odd. Since the centralizer $C_{G}(\sigma)$ contains $\langle\sigma\rangle$, we have $\# C_{G}(\sigma)=2^{n-1} \cdot 2^{b} \cdot m^{*}$, where $b \geq 0$ and $m^{*}$ is odd.

For $1 \leq j \leq l(\sigma)$, let $C_{j}$ be the conjugacy class in $G$ of $\sigma v_{j}$, and let $t\left(C_{j}\right) \in W G$ be the class sum of $C_{j}$. Since for all $j$, the centralizer $C_{G}\left(\sigma v_{j}\right)$ contains $\langle\sigma\rangle$, we have $\# C_{G}\left(\sigma v_{j}\right)=2^{n-1} \cdot 2^{b_{j}} \cdot m_{j}^{*}$, where $b_{j} \geq 0$ and $m_{j}^{*}$ is odd. We want to determine the action of $t\left(C_{j}\right)$ on $V_{\ell}$ for $2 \leq \ell \leq n-1$. For this, we identify $\operatorname{End}_{F G}\left(V_{\ell}\right) \cong F\left(\nu_{\ell}\right)$ with $\operatorname{End}_{F\left(\nu_{\ell}\right) G}\left(X_{\ell}\right)$ for one particular absolutely irreducible $F\left(\nu_{\ell}\right) G$-constituent $X_{\ell}$ of $V_{\ell}$ with character $\xi_{\ell}$. Using (5.10), we choose $\xi_{\ell}=\chi_{5,2^{n-1-\ell}}$. Then for $2 \leq \ell \leq n-1$, the action of $t\left(C_{j}\right)$ on $V_{\ell}$ is given as multiplication by $\mu_{j}(\ell)$, where

$$
\mu_{j}(\ell)=\frac{\# C_{j}}{\xi_{\ell}(1)} \cdot \xi_{\ell}\left(\sigma v_{j}\right)=2^{-b_{j}} \frac{g^{*}}{m_{j}^{*} \cdot x^{*}} \cdot \xi_{\ell}\left(\sigma v_{j}\right) .
$$

For $1 \leq j \leq l(\sigma)$, define $w_{j}=2^{b_{j}} \frac{m_{j}^{*} \cdot x^{*}}{g^{*}} \tilde{w}_{j}$. Then $w_{j} \in W$ since $g^{*}$ is odd, and $w_{j}$ does not depend on $\ell$. By (5.8) and (5.9),

$$
d_{\xi_{\ell}, \varphi^{(\sigma)}}^{(\sigma)}=d_{\chi_{5,2^{n-1-\ell}, \varphi^{(\sigma)}}^{(\sigma)}}^{(\sigma)} \nu_{\ell}
$$

Therefore, (5.12) and (5.13) imply that

$$
\nu_{\ell}=w_{1} \cdot \mu_{1}(\ell)+\cdots+w_{l(\sigma)} \cdot \mu_{l(\sigma)}(\ell)
$$

for certain $w_{1}, \ldots, w_{l(\sigma)} \in W$ which are independent of $\ell \in\{2, \ldots, n-1\}$.

Remark 5.2. If $B$ is a principal block, the above formulas (assuming $n \geq 3$ ) simplify considerably due to the fact that there is very little fusion of $D$-conjugacy classes in $G$ in this case. More precisely, following Brauer's arguments in [12, Sect. VII], suppose $D$ is a Sylow 2-subgroup of $G$ and let $S=\langle\sigma\rangle$. If $\sigma^{\lambda}$ is not in the center of $D$, then $S$ is a Sylow 2-subgroup of $C_{G}\left(\sigma^{\lambda}\right)$. Hence if $\sigma^{\lambda}$ and $\sigma^{\mu}$ are conjugate in $G$, we can use Sylow's theorems to see that they are conjugate in $N_{G}(S)$. Since $N_{G}(S) / C_{G}(S)$ is a 2-group, it must be of order 2 . Thus $N_{G}(S)$ is generated by $D$ and $C_{G}(S)$, which implies that $\sigma^{\lambda}$ and $\sigma^{\mu}$ are conjugate in $D$. Using (3.7) together with [15, Prop. (4A)] and [36, Prop. 2.10], we obtain that

$$
\chi_{5, i}(\sigma)=d_{\chi_{5, i}, \varphi^{(\sigma)}}^{(\sigma)} \varphi^{(\sigma)}\left(1_{G}\right),
$$

where $\varphi^{(\sigma)}$ is as in (5.8). Moreover, since $\varphi^{(\sigma)}$ is the unique Brauer character belonging to the block $b_{\sigma}$ of $k C_{G}(\sigma)$ satisfying $b_{\sigma}^{G}=B$, it follows by Brauer's Third Main Theorem (see e.g. [1, Thm. 16.1]) that $b_{\sigma}$ is the principal block of $k C_{G}(\sigma)$, which implies that $\varphi^{(\sigma)}$ is the trivial character of $b_{\sigma}$. Putting (5.15) into (5.13) for $j=1$ therefore implies that if $\omega=2^{b_{1}} \frac{m_{1}^{*} \cdot x^{*}}{g^{*}}$, which lies in $W$, then for all $2 \leq \ell \leq n-1$,

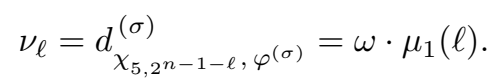

Note that in [2, Sect. 3.4], [3, Sect. 5] and [8, Sect. 3.2], it was assumed that formula (5.15) was also true for non-principal blocks. However, this may not be true since there could be more fusion of $D$-conjugacy classes in $G$ in this case. More precisely, let $Y$ be a full set of representatives of $D$-conjugacy classes of the elements of the set $\left\{\sigma^{r} \mid r\right.$ odd $\}$, and let $Y_{\sigma}$ be the set of all $y \in Y$ which are conjugate to $\sigma$ in $G$. If $B$ is not principal, then $\left|Y_{\sigma}\right|$ may be strictly greater than 1. By [15. Prop. (4A)] 
and [36, Prop. 2.10], the set $\left\{\left(y, b_{\sigma}\right) \mid y \in Y_{\sigma}\right\}$ is a system of representatives for the conjugacy classes of subsections for $B$ such that $y$ is conjugate to $\sigma$ in $G$. Hence it follows from Remark 3.1 that for non-principal blocks $B$, (5.15) has to be replaced by the formula

$$
\chi_{5, i}(\sigma)=\sum_{y \in Y_{\sigma}} d_{\chi_{5, i}, \varphi^{(\sigma)}}^{(y)} \varphi^{(\sigma)}\left(1_{G}\right) .
$$

Definition 5.3. Use the notation introduced above, and in particular (5.9). Assume $n \geq 3$.

(i) For $2 \leq \ell \leq n-1$, let min.pol. ${ }_{F}\left(\nu_{\ell}\right)(t) \in F[t]$ be the minimal polynomial of $\nu_{\ell}$ over $F$. Define

$$
q_{n}(t)=\prod_{\ell=2}^{n-1} \text { min.pol. }{ }_{F}\left(\nu_{\ell}\right)(t)
$$

and let $R^{\prime}=W[[t]] /\left(q_{n}(t)\right)$

(ii) Let $Z=\langle\sigma\rangle$, so that $Z$ is a cyclic group of order $2^{n-1}>2$. Let $\tau: Z \rightarrow Z$ be the group automorphism which sends $\sigma$ to $\sigma^{-1}$ if $D$ is dihedral or generalized quaternion, and which sends $\sigma$ to $\sigma^{-1+2^{n-2}}$ if $D$ is semidihedral. Then $\tau$ can be extended to a $W$-algebra automorphism of the group ring $W Z$ which will again be denoted by $\tau$. Let $T\left(\sigma^{2}\right)=1+\sigma^{2}+\sigma^{4}+\cdots+\sigma^{2^{n-1}}-2$, and define

$$
S^{\prime}=(W Z)^{\langle\tau\rangle} /\left(T\left(\sigma^{2}\right), \sigma T\left(\sigma^{2}\right)\right) .
$$

Remark 5.4. For $2 \leq \ell \leq n-1$, the minimal polynomial min.pol. ${ }_{F}\left(\nu_{\ell}\right)(t)$ is as follows:

$$
\begin{aligned}
& \min _{\text {.pol. }}\left(\nu_{2}\right)(t)=t, \\
& \text { min.pol. }{ }_{F}\left(\nu_{\ell}\right)(t)=\text { min.pol. }_{F}\left(\nu_{\ell-1}\right)\left(t^{2}-2\right) \quad \text { for } 3 \leq \ell \leq n-2 \text {, } \\
& \text { min.pol. }_{F}\left(\nu_{n-1}\right)(t)= \begin{cases}\min ^{\text {m.pol. }}{ }_{F}\left(\nu_{n-2}\right)\left(t^{2}-2\right) & \text { if } D \text { is dihedral or } \\
\text { min.pol. }_{F}\left(\nu_{n-2}\right)\left(t^{2}+2\right) & \text { generalized quaternion, } \\
\text { if } D \text { is semidihedral. }\end{cases}
\end{aligned}
$$

The $W$-algebra $R^{\prime}$ from Definition 5.3 is a complete local commutative Noetherian ring with residue field $k$. Moreover,

$$
\begin{aligned}
F \otimes_{W} R^{\prime} \cong \prod_{\ell=2}^{n-1} F\left(\nu_{\ell}\right) \quad \text { as } F \text {-algebras } \\
k \otimes_{W} R^{\prime} \cong k[t] /\left(t^{2^{n-2}-1}\right) \quad \text { as } k \text {-algebras. }
\end{aligned}
$$

Additionally, $R^{\prime}$ is isomorphic to the $W$-subalgebra of $\prod_{\ell=2}^{n-1} W\left[\nu_{\ell}\right]$ generated by the element $\left(\nu_{\ell}\right)_{\ell=2}^{n-1}$.

Lemma 5.5. Using the notation of Definition 5.3, there is a continuous $W$-algebra isomorphism $h: R^{\prime} \rightarrow S^{\prime}$ with $h(t)=\sigma+\tau(\sigma)$. In particular, $R^{\prime}$ is isomorphic to a subquotient algebra of the group algebra $W D$.

Moreover, if $D$ is dihedral or semidihedral, then the ring $W[[t]] /\left(t q_{n}(t), 2 q_{n}(t)\right)$ is also isomorphic to a subquotient algebra of the group algebra $W D$. 
Proof. If $D$ is dihedral or generalized quaternion, this follows from [2, Lemma 2.3.6] and [3, Lemma 5.3].

For the remainder of the proof, assume that $D$ is semidihedral (in particular, $n \geq 4)$. Let $J=\sigma^{2^{n-2}}$ so that $\tau(\sigma)=J \sigma^{-1}$. Note that $(W Z)^{\langle\tau\rangle}$ is generated as a $W$-algebra by $\left(\sigma+J \sigma^{-1}\right)$ and $J$. Moreover, $(W Z)^{\langle\tau\rangle}$ is a free $W$-module of rank $2^{n-2}+1$ with $W$-basis given by

$$
\begin{gathered}
\left(\sigma^{ \pm 1}+J \sigma^{\mp 1}\right),\left(\sigma^{ \pm 3}+J \sigma^{\mp 3}\right), \ldots,\left(\sigma^{ \pm\left(2^{n-3}-1\right)}+J \sigma^{\mp\left(2^{n-3}-1\right)}\right), \\
1, J,\left(\sigma^{2}+\sigma^{-2}\right),\left(\sigma^{4}+\sigma^{-4}\right), \ldots,\left(\sigma^{2^{n-2}-2}+\sigma^{-\left(2^{n-2}-2\right)}\right) .
\end{gathered}
$$

Note that the $W$-subalgebra of $(W Z)^{\langle\tau\rangle}$ generated by $\left(\sigma+J \sigma^{-1}\right)$ is a free $W$-module of the same rank $2^{n-2}+1$ and with almost the same $W$-basis as in (5.16) except that $J$ must be replaced by $2 J$. It follows that $S^{\prime}=(W Z)^{\langle\tau\rangle} /\left(T\left(\sigma^{2}\right), \sigma T\left(\sigma^{2}\right)\right)$ is generated as a $W$-algebra by the image of $\left(\sigma+J \sigma^{-1}\right)$ in $S^{\prime}$. Hence we have a surjective $W$-algebra homomorphism

$$
f: \quad W[[t]] \rightarrow S^{\prime}=(W Z)^{\langle\tau\rangle} /\left(T\left(\sigma^{2}\right), \sigma T\left(\sigma^{2}\right)\right)
$$

sending $t$ to the image in $S^{\prime}$ of $\sigma+J \sigma^{-1}=\sigma+\tau(\sigma)$. Using the injective $W$-algebra homomorphism

$$
\begin{aligned}
\iota: \quad W Z & \rightarrow W \times W \times \prod_{\ell=2}^{n-1} W\left[\zeta_{\ell}\right] \\
\sigma & \mapsto\left(1,-1,\left(\zeta_{\ell}\right)_{\ell=2}^{n-1}\right)
\end{aligned}
$$

it is straightforward to prove that there exists an odd integer $c_{n}$ such that $q_{n}\left(\sigma+J \sigma^{-1}\right)=c_{n} \sigma T\left(\sigma^{2}\right)$. More precisely, $c_{4}=3$ and $c_{n}=2 c_{n-1}^{2}-1$ for $n \geq 5$. Thus $q_{n}(t)$ lies in the kernel of $f$. Since both $R^{\prime}=W[[t]] /\left(q_{n}(t)\right)$ and $S^{\prime}$ are free as $W$-modules of the same rank $2^{n-2}-1$, it follows that $R^{\prime}=W[[t]] /\left(q_{n}(t)\right) \cong S^{\prime}$ as $W$-algebras.

To finish the proof of Lemma [5.5, it suffices to show that the ring $W[[t]] /$ $\left((t-2) q_{n}(t)\right)$ is isomorphic to a subquotient algebra of $W D$. Define

$$
\Theta=(W Z)^{\langle\tau\rangle} /\left(T\left(\sigma^{2}\right)-\sigma T\left(\sigma^{2}\right)\right) .
$$

Then $\Theta$ is isomorphic to a subquotient algebra of $W D$ and is generated as a $W$ algebra by the image of $\left(\sigma+J \sigma^{-1}\right)$ in $\Theta$. Moreover, $\Theta$ is a free $W$-module of rank $2^{n-2}$, since the ideal $\left(T\left(\sigma^{2}\right)-\sigma T\left(\sigma^{2}\right)\right)$ is generated over $W$ by $T\left(\sigma^{2}\right)-\sigma T\left(\sigma^{2}\right)$. Define a surjective $W$-algebra homomorphism $\theta: W[[t]] \rightarrow \Theta$ by sending $t$ to the image in $\Theta$ of $\sigma+J \sigma^{-1}=\sigma+\tau(\sigma)$. Using the above calculations, we see that

$$
\begin{aligned}
\theta\left((t-2) q_{n}(t)\right) & =\left(\left(\sigma+J \sigma^{-1}\right)-2\right) q_{n}\left(\sigma+J \sigma^{-1}\right) \\
& =\left(\left(\sigma+J \sigma^{-1}\right)-2\right) c_{n} \sigma T\left(\sigma^{2}\right) \\
& =2 c_{n}\left[T\left(\sigma^{2}\right)-\sigma T\left(\sigma^{2}\right)\right],
\end{aligned}
$$

which is zero in $\Theta$. Hence $(t-2) q_{n}(t)$ lies in the kernel of $\theta$. Since both $W[[t]] /$ $\left((t-2) q_{n}(t)\right)$ and $\Theta$ are free over $W$ of rank $2^{n-2}$, it follows that $W[[t]] /$ $\left((t-2) q_{n}(t)\right)$ is isomorphic to $\Theta$, which completes the proof of Lemma 5.5

The following result gives a correction of [3, Lemma 5.4] and generalizes the corrected result to all non-local blocks of infinite tame representation type. 
Lemma 5.6. Assume Hypothesis 4.1 and use the notation introduced above, in particular (5.9), (5.10) and Definition 5.3. Assume $n \geq 3$. If $D$ is quaternion of order 8 , let $U^{\prime}$ be a $W G$-module which is free over $W$ and whose $F$-character is either $\chi_{5,1}$, or $\chi_{6}$, or $\chi_{7}$. If $D$ is not quaternion of order 8 , let $U^{\prime}$ be a $W G$-module which is free over $W$ and whose $F$-character is equal to

$$
\sum_{\ell=2}^{n-1} \rho_{\ell}=\sum_{i=1}^{2^{n-2}-1} \chi_{5, i}
$$

(i) There exists a WG-module endomorphism $\alpha$ of $U^{\prime}$ such that the $W$-algebra $W[\alpha]$ generated by $\alpha$ is isomorphic to $R^{\prime}$.

(ii) Suppose either that $n=3$, or that $n \geq 4$ and $\operatorname{End}_{k G}\left(U^{\prime} / 2 U^{\prime}\right) \cong R^{\prime} / 2 R^{\prime}$ and $U^{\prime} / 2 U^{\prime}$ is free as a module for $\operatorname{End}_{k G}\left(U^{\prime} / 2 U^{\prime}\right)$ of rank $\operatorname{deg}\left(\chi_{5,1}\right)$. Then $\operatorname{End}_{W G}\left(U^{\prime}\right)=W[\alpha] \cong R^{\prime}$ and $U^{\prime}$ is free as a module for $\operatorname{End}_{W G}\left(U^{\prime}\right)$.

Proof. Suppose first that $n=3$. Then $q_{n}(t)=t$ and $R^{\prime} \cong W$. Hence we can choose the $W G$-module endomorphism $\alpha$ of $U^{\prime}$ to be the zero endomorphism. Since $U^{\prime}$ is free as a $W$-module and $F \otimes_{W} \operatorname{End}_{W G}\left(U^{\prime}\right) \cong \operatorname{End}_{F G}\left(F \otimes_{W} U^{\prime}\right) \cong F$, it follows that $\operatorname{End}_{W G}\left(U^{\prime}\right) \cong W \cong R^{\prime}$.

For the remainder of the proof, assume $n \geq 4$. We first construct a $W G$-module endomorphism $\alpha$ of $U^{\prime}$ as in part (i) of the lemma. As before, let $\sigma \in D$ be an element of order $2^{n-1}$ and let $\left\{v_{1}, \ldots, v_{l(\sigma)}\right\}$ be a complete system of representatives of $C_{G}(\sigma)$-conjugacy classes of 2-regular elements in $C_{G}(\sigma)$. For $1 \leq j \leq l(\sigma)$, let $C_{j}$ be the conjugacy class in $G$ of $\sigma v_{j}$, and let $t\left(C_{j}\right) \in W G$ be the class sum of $C_{j}$.

Let $1 \leq j \leq l(\sigma)$. Since $t\left(C_{j}\right)$ lies in the center of $W G$, multiplication by $t\left(C_{j}\right)$ defines a $W G$-module endomorphism of $U^{\prime}$. Since $U^{\prime}$ is free as a $W$-module, $\operatorname{End}_{W G}\left(U^{\prime}\right)$ can naturally be identified with a $W$-subalgebra of

$$
F \otimes_{W} \operatorname{End}_{W G}\left(U^{\prime}\right) \cong \operatorname{End}_{F G}\left(F \otimes_{W} U^{\prime}\right) \cong \prod_{\ell=2}^{n-1} \operatorname{End}_{F G}\left(V_{\ell}\right) \cong \prod_{\ell=2}^{n-1} F\left(\nu_{\ell}\right) .
$$

Therefore, $t\left(C_{j}\right)$ acts on $U^{\prime}$ as multiplication by a scalar $\lambda_{j}$ in the maximal $W$ order $\prod_{\ell=2}^{n-1} W\left[\nu_{\ell}\right]$ in $\prod_{\ell=2}^{n-1} F\left(\nu_{\ell}\right)$. Moreover, $\lambda_{j}$ can be determined from the action of $t\left(C_{j}\right)$ on $F \otimes_{W} U^{\prime} \cong \bigoplus_{\ell=2}^{n-1} V_{\ell}$, which implies by (5.13) that $\lambda_{j}=\left(\mu_{j}(\ell)\right)_{\ell=2}^{n-1}$. By (5.14), there exist elements $w_{1}, \ldots, w_{l(\sigma)} \in W$ such that

$$
\nu_{\ell}=w_{1} \cdot \mu_{1}(\ell)+\cdots+w_{l(\sigma)} \cdot \mu_{l(\sigma)}(\ell)
$$

for $2 \leq \ell \leq n-1$. Define $\alpha$ to be the $W G$-module endomorphism of $U^{\prime}$ given by multiplication by the element $\sum_{j=1}^{l(\sigma)} w_{j} t\left(C_{j}\right)$ in the center of $W G$. Then $\alpha$ acts on $U^{\prime}$ as multiplication by the scalar $\left(\nu_{\ell}\right)_{\ell=2}^{n-1} \in \prod_{\ell=2}^{n-1} W\left[\nu_{\ell}\right]$, which implies $W[\alpha] \cong R^{\prime}$ (see Remark 5.4).

Now let $\overline{U^{\prime}}=U^{\prime} / 2 U^{\prime}$ and suppose that $\operatorname{End}_{k G}\left(\overline{U^{\prime}}\right) \cong R^{\prime} / 2 R^{\prime} \cong k[t] /\left(t^{2^{n-2}-1}\right)$ and that $\overline{U^{\prime}}$ is free as a module for $\operatorname{End}_{k G}\left(U^{\prime} / 2 U^{\prime}\right)$ of rank $x=\operatorname{deg}\left(\chi_{5,1}\right)$. Note that $x=\operatorname{deg}\left(\chi_{5, i}\right)$ for all $1 \leq i \leq 2^{n-2}-1$ and that $\operatorname{dim}_{F\left(\nu_{\ell}\right)} V_{\ell}=x$ for all $2 \leq \ell \leq n-1$, which implies that $F \otimes_{W} U^{\prime}$ is a free module of $\operatorname{rank} x$ for $\operatorname{End}_{F G}\left(F \otimes_{W} U^{\prime}\right)$. We have a short exact sequence of $W$-modules:

$$
0 \longrightarrow \operatorname{End}_{W}\left(U^{\prime}\right) \stackrel{\cdot 2}{\longrightarrow} \operatorname{End}_{W}\left(U^{\prime}\right) \stackrel{\bmod 2}{\longrightarrow} \operatorname{End}_{k}\left(\overline{U^{\prime}}\right) \longrightarrow 0 .
$$


Considering the $G$-action on these modules, we obtain an exact sequence of $W$ modules:

$0 \longrightarrow \operatorname{End}_{W G}\left(U^{\prime}\right) \stackrel{\cdot 2}{\longrightarrow} \operatorname{End}_{W G}\left(U^{\prime}\right) \stackrel{\bmod 2}{\longrightarrow} \operatorname{End}_{k G}\left(\overline{U^{\prime}}\right) \longrightarrow \mathrm{H}^{1}\left(G, \operatorname{End}_{W}\left(U^{\prime}\right)\right)$.

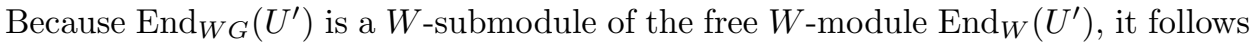
that $\operatorname{End}_{W G}\left(U^{\prime}\right)$ is a free $W$-module of rank

$$
\operatorname{dim}_{F}\left(F \otimes_{W} \operatorname{End}_{W G}\left(U^{\prime}\right)\right)=\operatorname{dim}_{F} \operatorname{End}_{F G}\left(F \otimes_{W} U^{\prime}\right)=2^{n-2}-1 .
$$

Since by assumption $\operatorname{dim}_{k} \operatorname{End}_{k G}\left(\overline{U^{\prime}}\right)=\operatorname{dim}_{k}\left(R^{\prime} / 2 R^{\prime}\right)=2^{n-2}-1$, it follows that (5.17) induces a short exact sequence of $W$-modules:

$$
0 \longrightarrow \operatorname{End}_{W G}\left(U^{\prime}\right) \stackrel{\cdot 2}{\longrightarrow} \operatorname{End}_{W G}\left(U^{\prime}\right) \stackrel{\bmod 2}{\longrightarrow} \operatorname{End}_{k G}\left(\overline{U^{\prime}}\right) \longrightarrow 0 .
$$

Let $\bar{\beta}$ be a generator of $\operatorname{End}_{k G}\left(\overline{U^{\prime}}\right)$ as a $k$-algebra. By $(\underline{5.18})$, there exists $\beta \in$ $\operatorname{End}_{W G}\left(U^{\prime}\right)$ whose induced $k G$-module endomorphism of $\overline{U^{\prime}}$ is equal to $\bar{\beta}$. Using Nakayama's lemma, we see that $\operatorname{End}_{W G}\left(U^{\prime}\right)=W[\beta]$. Since the maximal ideal $\mathfrak{m}_{W[\beta]}$ of $W[\beta]$ is generated by 2 and $\beta$ and the maximal ideal $\mathfrak{m}_{k[\bar{\beta}]}$ of $k[\bar{\beta}]$ is generated by $\bar{\beta}$, it follows that

$$
U^{\prime} / \mathfrak{m}_{W[\beta]} U^{\prime} \cong \overline{U^{\prime}} / \bar{\beta}\left(\overline{U^{\prime}}\right) \cong \overline{U^{\prime}} / \mathfrak{m}_{k[\bar{\beta}]} \overline{U^{\prime}},
$$

where the latter has $k$-dimension $x=\operatorname{deg}\left(\chi_{5,1}\right)$ by assumption. By Nakayama's lemma, we can lift a $k$-basis $\left\{\bar{s}_{1}, \ldots, \bar{s}_{x}\right\}$ of $U^{\prime} / \mathfrak{m}_{W[\beta]} U^{\prime}$ to a set $\left\{s_{1}, \ldots, s_{x}\right\}$ of generators of $U^{\prime}$ over $W[\beta]=\operatorname{End}_{W G}\left(U^{\prime}\right)$. Because $F \otimes_{W} U^{\prime}$ is a free module of rank $x$ for $\operatorname{End}_{F G}\left(F \otimes_{W} U^{\prime}\right) \cong F \otimes_{W} \operatorname{End}_{W G}\left(U^{\prime}\right)$, it follows that $s_{1}, \ldots, s_{x}$ are linearly independent over $\operatorname{End}_{W G}\left(U^{\prime}\right)$. Hence $U^{\prime}$ is free as a module for $\operatorname{End}_{W G}\left(U^{\prime}\right)$ of rank $x=\operatorname{deg}\left(\chi_{5,1}\right)$.

It remains to show that $\operatorname{End}_{W G}\left(U^{\prime}\right)=W[\alpha]$, i.e. we need to show $W[\alpha]=W[\beta]$. We identify both $W[\alpha]$ and $W[\beta]$ with $W$-subalgebras of the maximal $W$-order $\prod_{\ell=2}^{n-1} W\left[\nu_{\ell}\right]$ in $\prod_{\ell=2}^{n-1} F\left(\nu_{\ell}\right) \cong F \otimes_{W} \operatorname{End}_{W G}\left(U^{\prime}\right)$. Since $W[\alpha] \subseteq W[\beta]$, there exists a polynomial $q(X) \in W[X]$ such that $\alpha=q(\beta)$. Because $\alpha$ is not a unit, the constant coefficient of $q(X)$ must be divisible by 2 . Write

$$
q(X)=2 \tilde{a}_{0}+a_{1} X+a_{2} X^{2}+\cdots+a_{d} X^{d}
$$

for certain $\tilde{a}_{0}, a_{1}, \ldots, a_{d} \in W$ and $d \geq 1$. Suppose $a_{1}=2 \tilde{a}_{1}$ for some $\tilde{a}_{1} \in W$. Consider the natural projection

$$
\pi_{n-1}: \prod_{\ell=2}^{n-1} W\left[\nu_{\ell}\right] \rightarrow W\left[\nu_{n-1}\right],
$$

and let $\mathfrak{m}$ be the maximal ideal of $W\left[\nu_{n-1}\right]$. Since $n \geq 4$, either $\sqrt{2}$ or $\sqrt{-2}$ lies in $\mathfrak{m}$. Hence $2 \in \mathfrak{m}^{2}$. Since $2 \pi_{n-1}(\beta), \pi_{n-1}(\beta)^{2}, \ldots, \pi_{n-1}(\beta)^{d}$ all lie in $\mathfrak{m}^{2}$, it follows that $\pi_{n-1}(\alpha) \in \mathfrak{m}^{2}$. However, we have seen that $\pi_{n-1}(\alpha)=\nu_{n-1} \notin \mathfrak{m}^{2}$. Hence $a_{1}$ is not divisible by 2 . But then the $k G$-module endomorphism $\bar{\alpha}$ of $\overline{U^{\prime}}$ which is induced by $\alpha$ has the form

$$
\bar{\alpha}=\bar{a}_{1} \bar{\beta}+\bar{a}_{2} \bar{\beta}^{2}+\cdots+\bar{a}_{d} \bar{\beta}^{d},
$$

where $\bar{a}_{1} \in k^{*}$ and $\bar{a}_{2}, \ldots, \bar{a}_{d} \in k$. This means that $k[\bar{\alpha}]=k[\bar{\beta}]=\operatorname{End}_{k G}\left(\overline{U^{\prime}}\right)$, which implies by Nakayama's lemma that $\operatorname{End}_{W G}\left(U^{\prime}\right)=W[\alpha]$. 


\section{UNIVERSAL DEFORMATION RINGS}

Assume Hypothesis 4.1 and the notation introduced in Section 4 and Section 5. In particular, $p=2, n \geq 2$, and $D$ is dihedral, semidihedral or generalized quaternion. In this section, we want to determine $R(G, V)$ for any maximally ordinary $k G$-module $V$ in the sense of Definition 3.2 .

We first determine all indecomposable $k G$-modules $V$ belonging to $B$ whose stable endomorphism rings are isomorphic to $k$ and whose Brauer characters are restrictions of ordinary irreducible characters of height 1. By Remark 5.1, the ordinary irreducible characters of height 1 belonging to $B$ are (using the notation from Section 5):

- none if $n=2$,

- $\chi_{5,1}, \chi_{6}, \chi_{7}$ if $D$ is quaternion of order 8 , and

- $\chi_{5, i}$ for all $1 \leq i \leq 2^{n-2}-1$ if $n \geq 3$ and $D$ is not quaternion of order 8 .

Lemma 6.1. Assume Hypothesis 4.1, and assume $n \geq 3$. Let $\Lambda=k Q / I$ be a basic algebra such that $B$ is Morita equivalent to $\Lambda$. For each vertex $j$ in $Q$, let $T_{j}$ denote the simple $B$-module corresponding to the simple $\Lambda$-module belonging to $j$. Let $V$ be an indecomposable $k G$-module belonging to $B$ such that $\underline{\operatorname{End}}_{k G}(V) \cong k$ and such that the Brauer character of $V$ is equal to the restriction to the 2-regular conjugacy classes of an ordinary irreducible character of $G$ of height 1.

(a) Suppose $D$ is quaternion of order 8. If $Q=3 \mathcal{A}$, then $V$ is either isomorphic to $T_{1}$ or $T_{2}$, or $V$ is a uniserial module of length 4 whose radical quotient or socle is isomorphic to $T_{0}$. If $Q=3 \mathcal{K}$, then $V$ is an arbitrary uniserial module of length 2 .

(b) Suppose $D$ is not quaternion of order 8.

(i) If $Q=2 \mathcal{A}$, then $V$ is a uniserial module with descending composition factors $T_{0}, T_{0}, T_{1}$, or $T_{1}, T_{0}, T_{0}$.

(ii) If $Q=2 \mathcal{B}$ and $B$ is Morita equivalent to neither $\operatorname{SD}(2 \mathcal{B})_{4}(c)$ nor $\mathrm{Q}(2 \mathcal{B})_{2}(p, a, c)$, then $V$ is isomorphic to $T_{1}$. If $B$ is Morita equivalent to $\mathrm{SD}(2 \mathcal{B})_{4}(c)$ or $\mathrm{Q}(2 \mathcal{B})_{2}(p, a, c)$, then $V$ is a uniserial module with descending composition factors $T_{0}, T_{1}$, or $T_{1}, T_{0}$.

(iii) If $Q=3 \mathcal{A}$, then $V$ is a uniserial module with descending composition factors $T_{0}, T_{1}, T_{0}, T_{2}$, or $T_{2}, T_{0}, T_{1}, T_{0}$, or $T_{0}, T_{2}, T_{0}, T_{1}$, or $T_{1}, T_{0}, T_{2}, T_{0}$.

(iv) If $Q \in\{3 \mathcal{B}, 3 \mathcal{D}\}$, then $V$ is isomorphic to $T_{1}$.

(v) If $B$ is Morita equivalent to $\mathrm{SD}(3 \mathcal{C})_{2,1}$, then $V$ is isomorphic to $T_{0}$. If $B$ Morita equivalent to $\mathrm{SD}(3 \mathcal{C})_{2,2}$, then $V$ is indecomposable with descending radical factors $T_{0}, T_{1} \oplus T_{2}$, or $T_{1} \oplus T_{2}, T_{0}$, or $V$ is uniserial with descending composition factors $T_{1}, T_{0}, T_{2}$, or $T_{2}, T_{0}, T_{1}$.

(vi) If $Q \in\{3 \mathcal{H}, 3 \mathcal{K}\}$ and $B$ is not Morita equivalent to $\operatorname{SD}(3 \mathcal{H})_{2}$, then $V$ is a uniserial module with descending composition factors $T_{1}, T_{2}$, or $T_{2}, T_{1}$. If $B$ is Morita equivalent to $\mathrm{SD}(3 \mathcal{H})_{2}$, then $V$ is a uniserial module with descending composition factors $T_{0}, T_{1}$, or $T_{1}, T_{0}$.

Conversely, if $B$ and $V$ are as in $(a)$ or $(b)$, then $\operatorname{End}_{k G}(V) \cong k$ and the Brauer character of $V$ is equal to the restriction to the 2-regular conjugacy classes of an ordinary irreducible character of height 1 . Moreover, $\operatorname{Ext}_{k G}^{1}(V, V)=0$ if $D$ is quaternion of order 8 , and $\operatorname{Ext}_{k G}^{1}(V, V) \cong k$ in all other cases.

Proof. Lemma 6.1 is proved using the description of the basic algebra $\Lambda=k Q / I$ of the block $B$ together with its decomposition matrix as provided in Section 4 . 
To give an idea of the arguments, we discuss the case when $\Lambda=\operatorname{SD}(3 \mathcal{A})_{1}$ in part (b)(iii). It follows from the decomposition matrix in Figure 7 that $V$ is an indecomposable $B$-module with composition factors $T_{0}, T_{0}, T_{1}, T_{2}$. Let $t_{V}$ (resp. $s_{V}$ ) be the composition series length of the radical quotient (resp. socle) of $V$. If $t_{V}=3$, then $V$ has radical series length 2 and $s_{V}=1$. But then $V$ is a submodule of a projective indecomposable $B$-module which is impossible for $t_{V}=3$. If $t_{V}=2$, then $V$ has radical series length at most 3 and $s_{V} \leq 2$. Using that $\operatorname{Ext}_{B}^{1}\left(T_{i}, T_{j}\right)$ is one-dimensional over $k$ if $(i, j) \in\{(0,1),(0,2),(1,0),(2,0)\}$ and zero otherwise, it follows that there are no indecomposable $B$-modules $V$ with $t_{V}=2$ and radical series length 2. Analyzing $\operatorname{Ext}_{B}^{1}\left(T_{i_{1}} \oplus T_{i_{2}}, V^{\prime}\right)$ for appropriate indecomposable $B$ modules $V^{\prime}$ of length 2 , we see that the only indecomposable $B$-modules $V$ with $t_{V}=2$ and radical series length 3 are submodules of the projective cover $P_{T_{0}}$ of the form

\begin{tabular}{ccc}
$T_{0}$ & \multicolumn{2}{r}{$T_{0}$} \\
$T_{1} T_{2}$ & or & $T_{1} T_{2}$ \\
$T_{0}$ & & $T_{0}$
\end{tabular}.

However, the stable endomorphism ring of each of these modules has $k$-dimension 2. If $t_{V}=1$, then $V$ has radical series length at most 4 and $s_{V} \leq 3$. Since $V$ is a quotient module of a projective indecomposable $B$-module in this case, there are no indecomposable $B$-modules $V$ with $t_{V}=1$ and radical series length 2 . If $t_{V}=1$ and the radical series length of $V$ is 3 , we use similar Ext ${ }^{1}$ arguments as above to see that the only indecomposable $B$-modules $V$ with these properties are quotient modules of the projective cover $P_{T_{0}}$ with descending radical factors $T_{0}, T_{1} \oplus T_{2}, T_{0}$. But all such modules have an endomorphism which factors through $T_{0}$ and which does not factor through a projective module, meaning that the stable endomorphism ring of each of these modules has $k$-dimension 2. Finally, if $t_{V}=1$ and the radical series length of $V$ is 4 , then $V$ is one of the uniserial modules described in part (b)(iii) of Lemma 6.1. This description shows directly that $\operatorname{End}_{B}(V) \cong k$. Moreover, using the projective indecomposable $B$-modules, we see that $\operatorname{Ext}_{B}^{1}(V, V) \cong k$.

Corollary 6.2. Assume Hypothesis 4.1. Let $V$ be an indecomposable $k G$-module belonging to $B$ such that $\underline{\operatorname{End}}_{k G}(V) \cong k$. The following statements are equivalent:

(i) $V$ is maximally ordinary in the sense of Definition 3.2.

(ii) $n \geq 4$ and the Brauer character of $V$ is equal to the restriction to the 2regular conjugacy classes of an ordinary irreducible character of $G$ of height 1 ;

(iii) $n \geq 4$, and $B$ and $V$ are as in part (b) of Lemma 6.1,

Proof. If $n=2$ (resp. $n=3$ ), it follows from [14, Sect. VII] (resp. [15, 36]) that all generalized decomposition numbers corresponding to maximal 2-power order elements in $D$ lie in $\{0, \pm 1\}$. Hence there are no maximally ordinary $k G$-modules belonging to $B$ if $n \leq 3$.

Now suppose $n \geq 4$. Let $\sigma \in D$ be of maximal 2-power order, and let $\chi$ be an ordinary irreducible character belonging to $B$. It follows from [15, 36] that if $\chi$ has height 0 , then the non-zero generalized decomposition numbers corresponding to $\sigma$ and $\chi$ are \pm 1 . Also, if $\chi$ has height $n-2$, then all generalized decomposition numbers corresponding to $\sigma$ and $\chi$ are zero. On the other hand, we see from (5.8), since $n \geq 4$, that if $\chi$ has height 1 , then $d_{\chi, \varphi^{(\sigma)}}^{(\sigma)} \notin\{0, \pm 1\}$ if and only if $\chi=\chi_{5, i}$ 
for $i \in\left\{1,2, \ldots, 2^{n-2}-1\right\}-\left\{2^{n-3}\right\}$. Note that the restriction of $\chi_{5, i}$ to the 2 regular conjugacy classes is the same for all $1 \leq i \leq 2^{n-2}-1$. Therefore it follows that if $n \geq 4$, then $V$ is maximally ordinary if and only if its Brauer character is equal to the restriction to the 2-regular conjugacy classes of an ordinary irreducible character of height 1 . Hence Corollary 6.2 follows from Lemma 6.1.

The lifts of some of the $k G$-modules $V$ from Lemma 6.1 are connected to 3-tubes in the stable Auslander-Reiten quiver of $B$ as follows.

Definition 6.3. Assume Hypothesis 4.1, assume $n \geq 3$, and let $V$ be as in Lemma 6.1. We say $V$ corresponds to a 3-tube if there exists an indecomposable quotient module $\bar{U}$ of the projective $k G$-module cover $P_{V}$ of $V$ such that

(a) $\bar{U}$ defines a lift of $V$ over $k[t] /\left(t^{2^{n-2}}\right)$, and

(b) $\bar{U}$ belongs to a 3-tube of the stable Auslander-Reiten quiver of $B$.

The following lemma determines which modules $V$ from Lemma 6.1 correspond to 3 -tubes.

Lemma 6.4. Assume Hypothesis 4.1, assume $n \geq 3$, and let $V$ be as in Lemma 6.1. If $D$ is dihedral, then $V$ corresponds to a 3-tube, and if $D$ is generalized quaternion, then $V$ does not correspond to a 3-tube. If $D$ is semidihedral, then $V$ corresponds to a 3-tube if and only if either

- $B$ is Morita equivalent to one of the algebras in

$$
\left\{\mathrm{SD}(2 \mathcal{A})_{2}(c), \mathrm{SD}(2 \mathcal{B})_{1}(c), \mathrm{SD}(3 \mathcal{B})_{1}, \mathrm{SD}(3 \mathcal{C})_{2,1}\right\}
$$

and there are no further conditions on $V$; or

- $B$ is Morita equivalent to $\operatorname{SD}(3 \mathcal{A})_{1}$ and $V$ is such that its radical quotient or its socle is isomorphic to $T_{1}$; or

- $B$ is Morita equivalent to $\mathrm{SD}(3 \mathcal{C})_{2,2}$ and $V$ is such that its radical quotient or its socle is isomorphic to $T_{0}$; or

- $B$ is Morita equivalent to $\mathrm{SD}(3 \mathcal{H})_{1}$ and $V$ is such that its radical quotient is isomorphic to $T_{1}$;

- $B$ is Morita equivalent to $\mathrm{SD}(3 \mathcal{H})_{2}$ and $V$ is such that its radical quotient is isomorphic to $T_{0}$.

In all cases, if $V$ corresponds to a 3-tube, then $\bar{U}$ from Definition 6.3 belongs to the boundary of its 3-tube.

Proof. If $D$ is generalized quaternion, Lemma 6.4 follows from [27, V.4.3].

Suppose next that $D$ is dihedral, and let $\Lambda=k Q / I$ be a basic algebra such that $B$ is Morita equivalent to $\Lambda$. By [27, VI.10.1], it follows that $\Lambda / \operatorname{soc}(\Lambda)$ is special biserial. Therefore, we can use the techniques described in [17, Sect. 3] to determine the 3-tubes of the stable Auslander-Reiten quiver of $B$. Note that the modules at the boundaries of the 3 -tubes are either maximal uniserial or simple. Using the list of the possible $V$ in Lemma 6.1, we see by direct inspection that $V$ always corresponds to a 3 -tube and that the module $\bar{U}$ from Definition 6.3 always belongs to the boundary of its 3 -tube.

Finally, suppose that $D$ is semidihedral. By [27, V.4.2], the stable AuslanderReiten quiver of $B$ has at most one 3-tube. To give an idea of the arguments, we discuss the case when $B$ is Morita equivalent to $\Lambda=\operatorname{SD}(3 \mathcal{A})_{1}$. By Lemma 6.1(b)(iii), $V$ is a uniserial module with descending composition factors $T_{0}, T_{1}, T_{0}, T_{2}$, or 
$T_{2}, T_{0}, T_{1}, T_{0}$, or $T_{0}, T_{2}, T_{0}, T_{1}$, or $T_{1}, T_{0}, T_{2}, T_{0}$. Since $V$ is uniserial with $\operatorname{End}_{k G}(V)$ $\cong k \cong \operatorname{Ext}_{k G}^{1}(V, V)$, we can use [3, Lemma 2.5] to show that $R(G, V) / 2 R(G, V)$ is isomorphic to $k[t] /\left(t^{2^{n-2}}\right)$ (resp. $k[t] /\left(t^{2^{n-2}-1}\right)$ ) if the radical quotient or the socle of $V$ is isomorphic to $T_{1}$ (resp. $T_{2}$ ). Hence by Definition 6.3. $V$ can only correspond to a 3 -tube if the radical quotient or the socle of $V$ is isomorphic to $T_{1}$. Since the projective cover $P_{T_{1}}$ is uniserial, we obtain from [3, Lemma 2.5] that $\bar{U}=\Omega^{-1}\left(T_{1}\right)$ (resp. $\bar{U}=\Omega\left(T_{1}\right)$ ) defines a lift of $V$ over $k[t] /\left(t^{2^{n-2}}\right)$ if the radical quotient (resp. the socle) of $V$ is isomorphic to $T_{1}$. Since $\Omega^{3}(\bar{U}) \cong \bar{U}$, it follows that $\bar{U}$ belongs to a 3 -tube of the stable Auslander-Reiten quiver of $B$. Moreover, using the criterion given in [17, Sect. 1] for almost split sequences to have an indecomposable middle term, we see that $\bar{U}$ belongs to the boundary of this 3 -tube.

The following result shows that if $V$ corresponds to a 3-tube, then the module $\bar{U}$ from Definition 6.3 has a universal deformation $\operatorname{ring} R(G, \bar{U}) \cong k$.

Proposition 6.5. Assume Hypothesis 4.1, assume $n \geq 3$, and let $V$ be as in Lemma 6.1. Moreover, suppose that $V$ corresponds to a 3-tube. Let $\bar{U}$ be the $k G$-module from Definition 6.3 which belongs to a 3-tube of the stable Auslander-Reiten quiver of $B$. Then $\underline{\operatorname{End}}_{k G}(\bar{U}) \cong k$ and $R(G, \bar{U}) \cong k$.

Proof. If $D$ is dihedral, this follows from [2, Sect. 5.2] and [8, Prop. 6.3]. For the remainder of the proof, assume that $D$ is semidihedral.

Let $\mathfrak{T}$ be the 3-tube of the stable Auslander-Reiten quiver of $B$ to which $\bar{U}$ belongs. By Lemma 6.4 $\bar{U}$ belongs to the boundary of $\mathfrak{T}$. Going through the cases described in Lemma 6.4 it is straightforward to show that $\underline{\operatorname{End}}_{k G}(\bar{U}) \cong k$ and $\operatorname{Ext}_{k G}^{1}(\bar{U}, \bar{U})=0$.

Let $K$ be a vertex of $\bar{U}$. Because of the work in [27, Chap. V], and in particular [27. V.4.2.1 and proof of V.4.2], we have the following facts:

(i) The group $K$ is a Klein four group and the quotient group $N_{G}(K) / C_{G}(K)$ is isomorphic to a symmetric group $S_{3}$.

(ii) There is a block $b$ of $k N_{G}(K)$ with $b^{G}=B$ such that the Green correspondent $f \bar{U}$ of $\bar{U}$ belongs to the boundary of a 3-tube in the stable AuslanderReiten quiver of $b$. Moreover, $b$ is Morita equivalent to $k S_{4}$ modulo the socle.

Using these facts in lieu of [2, Facts 5.2.1], we can use similar arguments as in the proofs of [2, Prop. 5.2.4 and Cor. 5.2.5] to show that $R(G, \bar{U}) \cong k$.

By Example 3.4 and Corollary 6.2, Theorem 1.1] is a consequence of the following result.

Theorem 6.6. Assume Hypothesis 4.1, and assume $n \geq 3$. Let $V$ be an indecomposable $k G$-module belonging to $B$ such that $\underline{\operatorname{End}}_{k G}(V) \cong k$ and such that the Brauer character of $V$ is equal to the restriction to the 2-regular conjugacy classes of an ordinary irreducible character of $G$ of height 1 . Then

$$
R(G, V) \cong\left\{\begin{array}{cl}
W[[t]] /\left(t q_{n}(t), 2 q_{n}(t)\right) & \text { if } V \text { corresponds to a } 3 \text {-tube, } \\
W[[t]] /\left(q_{n}(t)\right) & \text { if } V \text { does not correspond to a } 3 \text {-tube, }
\end{array}\right.
$$

where $q_{n}(t)$ is as in Definition 5.3. In all cases, the ring $R(G, V)$ is a subquotient ring of $W D$, and it is a complete intersection ring if and only if $V$ does not correspond to a 3-tube. 
Proof. By Lemma 6.1, $V$ is one of the modules listed in part (a) or part (b) of that lemma.

Suppose first that $D$ is quaternion of order 8. By Lemma 6.1 (a), $\operatorname{Ext}_{k G}^{1}(V, V)=$ 0 , which implies that $R(G, V)$ is isomorphic to a quotient algebra of $W$. Using the decomposition matrices in Figures 8 and 18 together with [21, Prop. (23.7)], we see that in all cases $V$ can be lifted over $W$, which implies that $R(G, V) \cong W \cong$ $W[[t]] /\left(q_{3}(t)\right)$.

For the remainder of the proof, assume that $D$ is not quaternion of order 8 . In particular, the Brauer character of $V$ is the restriction of $\chi_{5,1}$ to the 2-regular conjugacy classes of $G$. By Lemma 6.1(b), $\operatorname{Ext}_{k G}^{1}(V, V) \cong k$, which implies that $R(G, V)$ is isomorphic to a quotient algebra of $W[[t]]$ but not to a quotient algebra of $W$. Let $P_{V}$ be the projective $k G$-module cover of $V$.

Claim 0. There exists an indecomposable quotient module $\overline{U^{\prime}}$ of $P_{V}$ such that $\overline{U^{\prime}}$ defines a lift of $V$ over $k[t] /\left(t^{2^{n-2}-1}\right)$ and $\overline{U^{\prime}} / t^{2} \overline{U^{\prime}}$ is an indecomposable $k G$-module. Moreover, if $V$ corresponds to a 3 -tube, then the indecomposable $k G$-module $\bar{U}$ of Definition 6.3. which is also an indecomposable quotient module of $P_{V}$, defines a lift of $V$ over $k[t] /\left(t^{2^{n-2}}\right)$ and satisfies $\bar{U} / t^{2^{n-2}-1} \bar{U} \cong \overline{U^{\prime}}$.

Proof of Claim 0. Suppose first that $B$ is Morita equivalent neither to $\operatorname{SD}(2 \mathcal{B})_{4}(c)$, nor to $\mathrm{Q}(2 \mathcal{B})_{2}(p, a, c)$, nor to $\mathrm{SD}(3 \mathcal{C})_{2,2}$. Then $V$ is uniserial of length $\ell \leq 4$. Using the quiver and relations of the basic algebra of $B$, as provided in Section 4 it follows that in all cases $P_{V}$ has an indecomposable quotient module $\overline{U^{\prime}}$ (resp. $\bar{U}$, provided it exists) which is uniserial of length $\ell\left(2^{n-2}-1\right)$ (resp. $\left.\ell 2^{n-2}\right)$. Moreover, $\overline{U^{\prime}}$ (resp. $\bar{U})$ can be pictured as having $2^{n-2}-1$ (resp. $2^{n-2}$ ) copies of $V$ stacked on top of each other. This means that the action of $t$ on $\overline{U^{\prime}}$ (resp. $\bar{U}$ ) is given by an automorphism of $\overline{U^{\prime}}$ (resp. $\bar{U}$ ) which is unique up to multiplication by a non-zero scalar and which factors through $\operatorname{rad}^{\ell}\left(\overline{U^{\prime}}\right)\left(\operatorname{resp} . \operatorname{rad}^{\ell}(\bar{U})\right)$. This implies Claim 0 in this case.

If $B$ is Morita equivalent to $\mathrm{SD}(2 \mathcal{B})_{4}(c)$ or $\mathrm{Q}(2 \mathcal{B})_{2}(p, a, c)$, it follows from Lemma 6.1(b)(ii) that $V$ is uniserial of length 2 with descending composition factors $T_{u}, T_{v}$, where $\{u, v\}=\{0,1\}$. Using the relations in Section 4.2, we see that the projective cover $P_{V}=P_{T_{u}}$ has a unique submodule $K_{u}$ which is uniserial with descending composition factors $T_{u}, T_{u}$. It follows that $\overline{U^{\prime}}=P_{T_{u}} / K_{u}$, which can be visualized as in (6.19), where $T_{u}$ (resp. $T_{v}$ ) occurs $2^{n-2}-1$ times. This implies Claim 0 in this case, since $V$ does not correspond to a 3 -tube, so $\bar{U}$ does not exist.

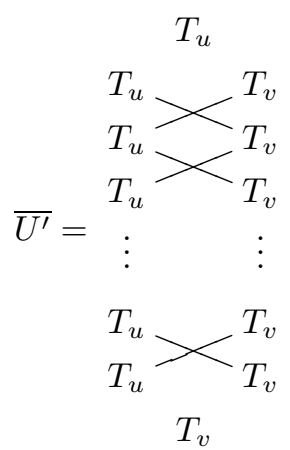

Finally, suppose $B$ is Morita equivalent to $\operatorname{SD}(3 \mathcal{C})_{2,2}$. By Lemma 6.1 (b)(v), $V$ is indecomposable with descending radical factors $T_{0}, T_{1} \oplus T_{2}$, or $T_{1} \oplus T_{2}, T_{0}$, or 
$V$ is uniserial with descending composition factors $T_{1}, T_{0}, T_{2}$, or $T_{2}, T_{0}, T_{1}$. Using the relations in Section 4.5. we see that there exists a unique uniserial $B$-module $T_{00}$, up to isomorphism, with descending composition factors $T_{0}, T_{0}$. Also, for $u \in\{1,2\}$, there exists a unique uniserial $B$-module $T_{u 0 u}$, up to isomorphism, with descending composition factors $T_{u}, T_{0}, T_{u}$. Moreover, the projective cover $P_{T_{0}}$ has a unique submodule isomorphic to $T_{00}$, and for $u \in\{1,2\}$, the projective cover $P_{T_{u}}$ has a unique submodule isomorphic to $T_{u 0 u}$. If the radical quotient (resp. socle) of $V$ is $T_{0}$, it follows that $\bar{U}$ is isomorphic to $\Omega^{-1}\left(T_{00}\right)\left(\operatorname{resp} . \Omega\left(T_{00}\right)\right)$, and $\overline{U^{\prime}}$ is isomorphic to $\operatorname{rad}^{2}(\bar{U})$. In particular, the action of $t$ on $\overline{U^{\prime}}(\operatorname{resp} . \bar{U})$ is given by an automorphism of $\overline{U^{\prime}}$ (resp. $\bar{U}$ ) which is unique up to multiplication by a non-zero scalar and which factors through $\operatorname{rad}^{2}\left(\overline{U^{\prime}}\right)\left(\operatorname{resp} \cdot \operatorname{rad}^{2}(\bar{U})\right)$. If the radical quotient of $V$ is $T_{u}$ for $u \in\{1,2\}$, it follows that $\bar{U}$ does not exist and $\overline{U^{\prime}}$ is isomorphic to $\Omega^{-1}\left(T_{u 0 u}\right)$. This completes the proof of Claim 0 .

Claim 1. The universal mod 2 deformation $\operatorname{ring} R(G, V) / 2 R(G, V)$ is isomorphic to $k[t] /\left(t^{2^{n-2}}\right)$ (resp. $\left.k[t] /\left(t^{2^{n-2}-1}\right)\right)$ and the universal $\bmod 2$ deformation of $V$ is isomorphic to $\bar{U}$ (resp. $\overline{U^{\prime}}$ ) if $V$ corresponds to a 3-tube (resp. does not correspond to a 3 -tube).

Proof of Claim 1. Suppose first that $B$ is Morita equivalent neither to $\operatorname{SD}(2 \mathcal{B})_{4}(c)$, nor to $\mathrm{Q}(2 \mathcal{B})_{2}(p, a, c)$, nor to $\mathrm{SD}(3 \mathcal{C})_{2,2}$. As seen in the proof of Claim $0, V$ is uniserial in this case. Moreover, it is straightforward to check that the projective cover of $V$ satisfies the hypotheses of [3, Lemma 2.5]. Hence we can use [3, Lemma $2.5]$ to prove Claim 1 in this case.

If $B$ is Morita equivalent to $\mathrm{SD}(2 \mathcal{B})_{4}(c)$ or $\mathrm{Q}(2 \mathcal{B})_{2}(p, a, c)$, it follows from Lemma 6.1(b)(ii) that $V$ is uniserial with descending composition factors $T_{u}, T_{v}$ where $\{u, v\}=\{0,1\}$. As seen in the proof of Claim 0 , the projective cover $P_{V}=P_{T_{u}}$ has a unique submodule $K_{u}$ which is uniserial with descending composition factors $T_{u}, T_{u}$, and $\overline{U^{\prime}}=P_{T_{u}} / K_{u}$ can be visualized as in (6.19). Using the relations in Section 4.2, we see that there is a unique $B$-submodule $V^{\prime}$ of $\overline{U^{\prime}}$ which is isomorphic to $V$. Moreover, if $T=\overline{U^{\prime}} / V^{\prime}$, then there is a unique $B$-submodule $T^{\prime}$ of $\overline{U^{\prime}}$ such that there are $k G$-module isomorphisms $\varphi: \overline{U^{\prime}} / T^{\prime} \rightarrow V$ and $\psi: \overline{U^{\prime}} / V^{\prime}=T \rightarrow T^{\prime}$. Since $\operatorname{Ext}_{k G}^{1}\left(\overline{U^{\prime}}, V\right)=0$ and since every surjective $k G$-module homomorphism $\overline{U^{\prime}} \rightarrow V$ must have kernel equal to $T^{\prime}$, we can argue as in the proof of [3, Lemma 2.5] to show that $R(G, V) / 2 R(G, V)$ is isomorphic to $k[t] /\left(t^{2^{n-2}-1}\right)$ and that the universal mod 2 deformation of $V$ is isomorphic to $\overline{U^{\prime}}$. This implies Claim 1 in this case.

If $B$ is Morita equivalent to $\operatorname{SD}(3 \mathcal{C})_{2,2}$, we again use similar arguments as in the proof of [3. Lemma 2.5] to prove Claim 1. The main point is that, as seen in the proof of Claim $0, \overline{U^{\prime}}$ and $\bar{U}$ are suitable submodules (resp. suitable quotient modules) of the projective cover $P_{T_{0}}$ if the radical quotient (resp. socle) of $V$ is $T_{0}$, and $\overline{U^{\prime}}$ is a suitable quotient module of $P_{T_{u}}$ if the radical quotient of $V$ is $T_{u}$ for $u \in\{1,2\}$. This completes the proof of Claim 1 .

Claim 2. In all cases, $\operatorname{End}_{k G}\left(\overline{U^{\prime}}\right) \cong k[t] /\left(t^{2^{n-2}-1}\right)$.

Proof of Claim 2. Suppose first that $B$ is Morita equivalent neither to $\operatorname{SD}(2 \mathcal{B})_{4}(c)$, nor to $\mathrm{Q}(2 \mathcal{B})_{2}(p, a, c)$, nor to $\operatorname{SD}(3 \mathcal{C})_{2,2}$. As seen in the proof of Claim $0, \overline{U^{\prime}}$ is a uniserial module, which can be pictured as having $2^{n-2}-1$ copies of $V$ stacked on top of each other. Since $\overline{U^{\prime}}$ is isomorphic to a tree module in the sense of [32], we can use the main result of [32] to prove Claim 2. 
If $B$ is Morita equivalent to $\Lambda \in\left\{\mathrm{SD}(2 \mathcal{B})_{4}(c), \mathrm{Q}(2 \mathcal{B})_{2}(p, a, c), \mathrm{SD}(3 \mathcal{C})_{2,2}\right\}$, we use the relations in Section 4.2 and Section 4.5 to analyze the possible $k G$-module endomorphisms of $\overline{U^{\prime}}$, where we use the description of $\overline{U^{\prime}}$ as given in the proof of Claim 0. Using explicit $k$-bases for the $\Lambda$-module $\overline{U_{\Lambda}^{\prime}}$ corresponding to $\overline{U^{\prime}}$ under the Morita equivalence, a straightforward linear algebra calculation shows that $\operatorname{End}_{\Lambda}\left(\overline{U_{\Lambda}^{\prime}}\right) \cong k[t] /\left(t^{2^{n-2}-1}\right)$. This proves Claim 2 .

Claim 3. In all cases, $\overline{U^{\prime}}$ has a lift $U^{\prime}$ over $W$ such that the $F$-character of $U^{\prime}$ is equal to

where $\rho_{\ell}$ is as in (5.10).

$$
\sum_{\ell=2}^{n-1} \rho_{\ell}=\sum_{i=1}^{2^{n-2}-1} \chi_{5, i}
$$

Proof of Claim 3. In all cases, we use the description of $\overline{U^{\prime}}$ as given in the proof of Claim 0. Suppose first that either $B$ is not Morita equivalent to any of the algebras in

$$
\left\{\mathrm{SD}(2 \mathcal{A})_{1}(c), \mathrm{Q}(2 \mathcal{A})(c), \mathrm{SD}(3 \mathcal{A})_{1}, \mathrm{Q}(3 \mathcal{A})_{2}\right\}
$$

or $B$ is Morita equivalent to $\operatorname{SD}(3 \mathcal{A})_{1}$ and $V$ is such that its radical quotient (resp. socle) is isomorphic to $T_{1}$. Then Claim 3 follows by using the decomposition matrix of $B$ together with [21, Prop. (23.7)].

Suppose next that $B$ is Morita equivalent to one of the algebras in $\left\{\operatorname{SD}(2 \mathcal{A})_{1}(c)\right.$, $\mathrm{Q}(2 \mathcal{A})(c)\}$. If the radical quotient (resp. socle) of $V$ is isomorphic to $T_{1}$, then $\Omega\left(\overline{U^{\prime}}\right) \cong Z_{001}\left(\right.$ resp. $\left.\Omega^{-1}\left(\overline{U^{\prime}}\right) \cong Z_{100}\right)$, where

$$
Z_{001}=\begin{gathered}
T_{1} \\
T_{1} T_{0} \\
T_{0} \\
T_{1}
\end{gathered} \quad Z_{100}=\begin{gathered}
T_{1} \\
T_{1} T_{0} \\
\quad T_{1}
\end{gathered}
$$

and $Z_{001}$ is a submodule of $P_{T_{1}}$ and $Z_{100}$ is a quotient module of $P_{T_{1}}$. Using [2. Lemma 2.3.2], it follows that $Z_{001}$ and $Z_{100}$ each have a lift over $W$ such that the $F$-character of this lift is $\chi_{3}+\chi_{4}+\chi_{6}$ (using the notation in Figure 2), which implies Claim 3 in this case.

Finally, suppose that $B$ is Morita equivalent to $\operatorname{SD}(3 \mathcal{A})_{1}$ and $u=2$ and $v=1$, or $B$ is Morita equivalent to $\mathrm{Q}(3 \mathcal{A})_{2}$ and $\{u, v\}=\{1,2\}$. If $V$ is such that its radical quotient (resp. socle) is isomorphic to $T_{u}$, then $\Omega\left(\overline{U^{\prime}}\right)=Z_{0 v 0 u}\left(\operatorname{resp} . \Omega^{-1}\left(\overline{U^{\prime}}\right)=\right.$ $\left.Z_{u 0 v 0}\right)$, where

$$
\begin{aligned}
& T_{u} T_{u} \\
& T_{0} T_{0} \\
& \begin{array}{c}
Z_{0 v 0 u}=T_{u} T_{v} \quad, \quad Z_{u 0 v 0}=T_{u} T_{v} \\
T_{0} \\
T_{u}
\end{array}
\end{aligned}
$$

and $Z_{0 v 0 u}$ is a submodule of $P_{T_{u}}$ and $Z_{u 0 v 0}$ is a quotient module of $P_{T_{u}}$. Using [2, Lemma 2.3.2], it follows that $Z_{0 v 0 u}$ and $Z_{u 0 v 0}$ each have a lift over $W$ such that the $F$-character of this lift is $\chi_{2}+\chi_{3}+\chi_{6}$ if $B$ is Morita equivalent to $\operatorname{SD}(3 \mathcal{A})_{1}$ (using the notation in Figure 7) and the $F$-character of this lift is $\chi_{2}+\chi_{4}+\chi_{6}$ (resp. $\left.\chi_{2}+\chi_{3}+\chi_{7}\right)$ if $B$ is Morita equivalent to $\mathrm{Q}(3 \mathcal{A})_{2}$ and $u=1$ (resp. $u=2$ ) (using the notation in Figure 8). This completes the proof of Claim 3. 
Claim 4. The universal deformation ring $R(G, V)$ is as stated in Theorem 6.6.

Proof of Claim 4. In all cases, it follows by Lemma [5.6 that $U^{\prime}$ from Claim 3 is an $R^{\prime} G$-module. More precisely, there exists a $W G$-module endomorphism $\alpha$ of $U^{\prime}$ such that $W[\alpha] \cong R^{\prime}$. By Claim 2 , we have $\operatorname{End}_{k G}\left(\overline{U^{\prime}}\right) \cong k[t] /\left(t^{2^{n-2}-1}\right) \cong$ $R^{\prime} / 2 R^{\prime}$. Moreover, since $\overline{U^{\prime}}$ is a lift of $V$ over $k[t] /\left(t^{2^{n-2}-1}\right), \overline{U^{\prime}}$ is free as a module for $\operatorname{End}_{k G}\left(\overline{U^{\prime}}\right)$ of $\operatorname{rank} \operatorname{dim}_{k} V=\operatorname{deg}\left(\chi_{5,1}\right)$. Hence it follows by Lemma 5.6 that $\operatorname{End}_{W G}\left(U^{\prime}\right)=W[\alpha] \cong R^{\prime}$ and $U^{\prime}$ is free as a module for $\operatorname{End}_{W G}\left(U^{\prime}\right)$.

In other words, $U^{\prime}$ defines a lift of $V$ over $R^{\prime}$. Let $\tau: R(G, V) \rightarrow R^{\prime}$ be the unique continuous $W$-algebra homomorphism relative to the deformation defined by $U^{\prime}$. Since $R^{\prime} /\left(\mathfrak{m}_{R^{\prime}}^{2}+2 R^{\prime}\right) \cong k[t] /\left(t^{2}\right), \tau$ is surjective if and only if $R^{\prime} /\left(\mathfrak{m}_{R^{\prime}}^{2}+2 R^{\prime}\right) \otimes_{R^{\prime}} U^{\prime}$ does not define the trivial lift of $V$ over $k[t] /\left(t^{2}\right)$. However,

$$
R^{\prime} /\left(\mathfrak{m}_{R^{\prime}}^{2}+2 R^{\prime}\right) \otimes_{R^{\prime}} U^{\prime} \cong U^{\prime} /\left(\alpha^{2}\left(U^{\prime}\right)+2 U^{\prime}\right) \cong \overline{U^{\prime}} / \bar{\alpha}^{2}\left(\overline{U^{\prime}}\right) \cong \overline{U^{\prime}} / t^{2} \overline{U^{\prime}}
$$

is an indecomposable $k G$-module, which implies that this does not define the trivial lift of $V$ over $k[t] /\left(t^{2}\right)$. Hence $\tau$ is surjective and induces a surjective $k$-algebra homomorphism

$$
\bar{\tau}: R(G, V) / 2 R(G, V) \rightarrow R^{\prime} / 2 R^{\prime} .
$$

Suppose first that $V$ does not correspond to a 3-tube. Then $R(G, V) / 2 R(G, V)$ and $R^{\prime} / 2 R^{\prime}$ are isomorphic and finite-dimensional over $k$, which implies that $\bar{\tau}$ is an isomorphism. Because $R^{\prime}$ is a free $W$-module of finite rank, it follows that $\tau$ is an isomorphism. By Lemma 5.5 $R^{\prime}$ is isomorphic to a subquotient ring of $W D$. This proves Claim 4 and completes the proof of Theorem 6.6 if $V$ does not correspond to a 3 -tube.

Suppose next that $V$ corresponds to a 3 -tube. Then the universal mod 2 deformation ring $R(G, V) / 2 R(G, V)$ is isomorphic to $k[t] /\left(2^{n-2}\right)$ and the universal mod 2 deformation is given by the isomorphism class of $\bar{U}$. By [2, Lemma 2.3.3], it follows that $R(G, V) \cong W[[t]] /\left(q_{n}(t)(t-2 \mu), a 2^{m} q_{n}(t)\right)$ for certain $\mu \in W, a \in\{0,1\}$ and $0<m \in \mathbb{Z}$. If $a=0$, then $R(G, V) \cong W[[t]] /\left(q_{n}(t)(t-2 \mu)\right)$ is free over $W$. If $a=1$, then $R(G, V) / 2^{m} R(G, V) \cong\left(W / 2^{m} W\right)[[t]] /\left(q_{n}(t)(t-2 \mu)\right)$ is free over $W / 2^{m} W$. Therefore it follows that if $a=0$ (resp. $a=1$ ), then there is a lift of $\bar{U}$, when regarded as a $k G$-module, over $W$ (resp. over $W / 2^{m} W$ ). But by Proposition 6.5 we have $R(G, \bar{U}) \cong k$, which means we must have $a=1$ and $m=1$. Since $V$ corresponds to a 3-tube, $D$ is dihedral or semidihedral. Hence by Lemma 5.5 the ring $W[[t]] /\left(t q_{n}(t), 2 q_{n}(t)\right)$ is isomorphic to a subquotient ring of $W D$. This proves Claim 4 and completes the proof of Theorem 6.6 if $V$ corresponds to a 3-tube.

Appendix: Decomposition matrices for the algebras in Section 4

$$
\begin{gathered}
\varphi_{0} \varphi_{1} \\
\chi_{1} \\
\chi_{2} \\
\chi_{3} \\
\chi_{4} \\
\chi_{5, i}
\end{gathered} \quad\left[\begin{array}{ll}
1 & 0 \\
1 & 0 \\
1 & 1 \\
1 & 1 \\
2 & 1
\end{array}\right] \quad 1 \leq i \leq 2^{n-2}-1
$$

FIgURE 1. The decomposition matrix for blocks of type $\mathrm{D}(2 \mathcal{A})$ or $\operatorname{SD}(2 \mathcal{A})_{2}(c)$. 


$$
\begin{gathered}
\varphi_{0} \varphi_{1} \\
\chi_{1} \\
\chi_{2} \\
\chi_{3} \\
\chi_{4} \\
\chi_{5, i} \\
\chi_{6}
\end{gathered} \quad\left[\begin{array}{ll}
1 & 0 \\
1 & 0 \\
1 & 1 \\
1 & 1 \\
2 & 1 \\
0 & 1
\end{array}\right] \quad 1 \leq i \leq 2^{n-2}-1
$$

Figure 2. The decomposition matrix for blocks of type $\mathrm{SD}(2 \mathcal{A})_{1}(c)$ or $\mathrm{Q}(2 \mathcal{A})(c)$.

$$
\begin{gathered}
\varphi_{0} \varphi_{1} \\
\chi_{1} \\
\chi_{2} \\
\chi_{3} \\
\chi_{4} \\
\chi_{5, i}
\end{gathered} \quad\left[\begin{array}{ll}
1 & 0 \\
1 & 0 \\
1 & 1 \\
1 & 1 \\
0 & 1
\end{array}\right] \quad 1 \leq i \leq 2^{n-2}-1
$$

FIGURE 3. The decomposition matrix for blocks of type $\mathrm{D}(2 \mathcal{B})$ or $\operatorname{SD}(2 \mathcal{B})_{1}(c)$.

$$
\begin{gathered}
\varphi_{0} \varphi_{1} \\
\chi_{1} \\
\chi_{2} \\
\chi_{3} \\
\chi_{4} \\
\chi_{5, i} \\
\chi_{6}
\end{gathered} \quad\left[\begin{array}{ll}
1 & 0 \\
1 & 0 \\
1 & 1 \\
1 & 1 \\
0 & 1 \\
2 & 1
\end{array}\right] \quad 1 \leq i \leq 2^{n-2}-1
$$

Figure 4. The decomposition matrix for blocks of type $\mathrm{SD}(2 \mathcal{B})_{2}(c)$ or $\mathrm{Q}(2 \mathcal{B})_{1}(c)$.

$$
\begin{gathered}
\varphi_{0} \varphi_{1} \\
\chi_{1} \\
\chi_{2} \\
\chi_{3} \\
\chi_{4} \\
\chi_{5, i}
\end{gathered} \quad\left[\begin{array}{ll}
1 & 0 \\
1 & 0 \\
0 & 1 \\
0 & 1 \\
1 & 1
\end{array}\right] \quad 1 \leq i \leq 2^{n-2}-1
$$

Figure 5. The decomposition matrix for blocks of type $\mathrm{SD}(2 \mathcal{B})_{4}(c)$ or $\mathrm{Q}(2 \mathcal{B})_{2}(p, a, c)$. 


$\begin{gathered}\varphi_{0} \varphi_{1} \varphi_{2} \\ \chi_{1} \\ \chi_{2} \\ \chi_{3} \\ \chi_{4} \\ \chi_{5, i}\end{gathered} \quad\left[\begin{array}{lll}1 & 0 & 0 \\ 1 & 1 & 1 \\ 1 & 0 & 1 \\ 1 & 1 & 0 \\ 2 & 1 & 1\end{array}\right] \quad 1 \leq i \leq 2^{n-2}-1$

Figure 6 . The decomposition matrix for blocks of type $\mathrm{D}(3 \mathcal{A})_{1}$.

$\begin{gathered}\varphi_{0} \varphi_{1} \varphi_{2} \\ \chi_{1} \\ \chi_{2} \\ \chi_{3} \\ \chi_{4} \\ \chi_{5, i} \\ \chi_{6}\end{gathered} \quad\left[\begin{array}{lll}1 & 0 & 0 \\ 1 & 1 & 1 \\ 1 & 0 & 1 \\ 1 & 1 & 0 \\ 2 & 1 & 1 \\ 0 & 0 & 1\end{array}\right] \quad 1 \leq i \leq 2^{n-2}-1$

Figure 7. The decomposition matrix for blocks of type $\operatorname{SD}(3 \mathcal{A})_{1}$.

$\begin{gathered}\varphi_{0} \varphi_{1} \varphi_{2} \\ \chi_{1} \\ \chi_{2} \\ \chi_{3} \\ \chi_{4} \\ \chi_{5, i} \\ \chi_{6} \\ \chi_{7}\end{gathered} \quad\left[\begin{array}{lll}1 & 0 & 0 \\ 1 & 1 & 1 \\ 1 & 0 & 1 \\ 1 & 1 & 0 \\ 2 & 1 & 1 \\ 0 & 1 & 0 \\ 0 & 0 & 1\end{array}\right] \quad 1 \leq i \leq 2^{n-2}-1$

FiguRE 8. The decomposition matrix for blocks of type $\mathrm{Q}(3 \mathcal{A})_{2}$.

$$
\begin{gathered}
\varphi_{0} \varphi_{1} \\
\begin{array}{c}
\varphi_{2} \\
\chi_{1} \\
\chi_{2} \\
\chi_{3} \\
\chi_{4} \\
\chi_{5, i}
\end{array} \quad\left[\begin{array}{lll}
1 & 0 & 0 \\
1 & 1 & 0 \\
1 & 0 & 1 \\
1 & 1 & 1 \\
0 & 1 & 0
\end{array}\right] \quad 1 \leq i \leq 2^{n-2}-1
\end{gathered}
$$

FiguRE 9. The decomposition matrix for blocks of type $\mathrm{D}(3 \mathcal{B})_{1}$. 


\begin{tabular}{|c|c|c|c|}
\hline \multicolumn{3}{|c|}{$\varphi_{0} \varphi_{1} \varphi_{2}$} & \\
\hline$\chi_{1}$ & 1 & 0 & \\
\hline$\chi_{2}$ & & 0 & \\
\hline$\chi_{3}$ & 1 & 1 & \\
\hline$\chi_{4}$ & & 1 & \\
\hline$\chi_{5, i}$ & 0 & 0 & $1 \leq i \leq 2^{n-2}-1$ \\
\hline$\chi_{6}$ & 0 & 1 & \\
\hline
\end{tabular}

Figure 10. The decomposition matrix for blocks of type $\operatorname{SD}(3 \mathcal{B})_{1}$ or $\mathrm{SD}(3 \mathcal{D})$.

$\begin{gathered}\varphi_{0} \\ \varphi_{1} \\ \chi_{1} \\ \chi_{2} \\ \chi_{3} \\ \chi_{4} \\ \chi_{5, i} \\ \chi_{6}\end{gathered} \quad\left[\begin{array}{lll}1 & 0 & 0 \\ 1 & 1 & 0 \\ 1 & 1 & 1 \\ 1 & 0 & 1 \\ 0 & 1 & 0 \\ 2 & 1 & 1\end{array}\right] \quad 1 \leq i \leq 2^{n-2}-1$

Figure 11. The decomposition matrix for blocks of type $\operatorname{SD}(3 \mathcal{B})_{2}$.

$\begin{gathered}\varphi_{0} \varphi_{1} \varphi_{2} \\ \chi_{1} \\ \chi_{2} \\ \chi_{3} \\ \chi_{4} \\ \chi_{5, i} \\ \chi_{6} \\ \chi_{7}\end{gathered} \quad\left[\begin{array}{lll}1 & 0 & 0 \\ 1 & 1 & 0 \\ 1 & 1 & 1 \\ 1 & 0 & 1 \\ 0 & 1 & 0 \\ 0 & 0 & 1 \\ 2 & 1 & 1\end{array}\right] \quad 1 \leq i \leq 2^{n-2}-1$

Figure 12. The decomposition matrix for blocks of type $\mathrm{Q}(3 \mathcal{B})$.

$$
\begin{aligned}
& \varphi_{0} \varphi_{1} \varphi_{2} \\
& \begin{array}{c}
\chi_{1} \\
\chi_{2} \\
\chi_{3} \\
\chi_{4} \\
\chi_{5, i} \\
\chi_{6}
\end{array} \quad\left[\begin{array}{lll}
0 & 1 & 0 \\
1 & 1 & 0 \\
1 & 0 & 1 \\
0 & 0 & 1 \\
1 & 0 & 0 \\
1 & 1 & 1
\end{array}\right] \quad 1 \leq i \leq 2^{n-2}-1
\end{aligned}
$$

FIgURE 13. The decomposition matrix for blocks of type $\operatorname{SD}(3 \mathcal{C})_{2,1}$. 


\begin{tabular}{|c|c|c|c|}
\hline \multicolumn{3}{|c|}{$\varphi_{0} \varphi_{1} \varphi_{2}$} & \\
\hline$\chi_{1}$ & $\begin{array}{ll}0 & 1\end{array}$ & 0 & \\
\hline$\chi_{2}$ & 10 & 1 & \\
\hline$\chi_{3}$ & 11 & 0 & \\
\hline$\chi_{4}$ & $\begin{array}{ll}0 & 0\end{array}$ & 1 & \\
\hline$\chi_{5, i}$ & 11 & 1 & $1 \leq i \leq 2^{n-2}-1$ \\
\hline$\chi_{6}$ & 10 & 0 & \\
\hline
\end{tabular}

Figure 14. The decomposition matrix for blocks of type $\operatorname{SD}(3 \mathcal{C})_{2,2}$.

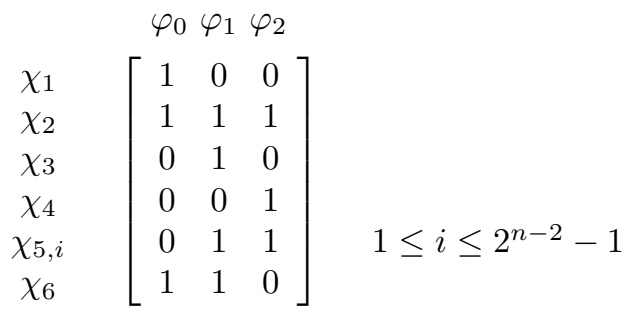

Figure 15. The decomposition matrix for blocks of type $\operatorname{SD}(3 \mathcal{H})_{1}$.

\begin{tabular}{|c|c|c|c|}
\hline & $\varphi_{0} 4$ & $\rho_{1} \varphi$ & \\
\hline$\chi_{1}$ & 1 & 0 & \\
\hline$\chi_{2}$ & 0 & 1 & \\
\hline$\chi_{3}$ & & 1 & \\
\hline$\chi_{4}$ & 0 & 0 & \\
\hline$\chi_{5, i}$ & 1 & 1 & $1 \leq i \leq 2^{n-2}-1$ \\
\hline$\chi_{6}$ & 0 & 1 & \\
\hline
\end{tabular}

Figure 16. The decomposition matrix for blocks of type $\operatorname{SD}(3 \mathcal{H})_{2}$.

$\varphi_{0} \varphi_{1}$
$\begin{gathered}\varphi_{2} \\ \chi_{1} \\ \chi_{2} \\ \chi_{3} \\ \chi_{4} \\ \chi_{5, i}\end{gathered} \quad\left[\begin{array}{lll}1 & 0 & 0 \\ 1 & 1 & 1 \\ 0 & 1 & 0 \\ 0 & 0 & 1 \\ 0 & 1 & 1\end{array}\right] \quad 1 \leq i \leq 2^{n-2}-1$

Figure 17. The decomposition matrix for blocks of type $\mathrm{D}(3 \mathcal{K})$. 


$\begin{gathered}\chi_{1} \\ \chi_{2} \\ \chi_{3} \\ \chi_{4} \\ \chi_{5, i} \\ \chi_{6} \\ \chi_{7}\end{gathered} \quad\left[\begin{array}{lll}1 & 0 & 0 \\ 1 & 1 & 1 \\ 0 & 1 & 0 \\ 0 & 0 & 1 \\ 0 & 1 & 1 \\ 1 & 0 & 1 \\ 1 & 1 & 0\end{array}\right] \quad 1 \leq i \leq 2^{n-2}-1$

Figure 18. The decomposition matrix for blocks of type $\mathrm{Q}(3 \mathcal{K})$.

\section{REFERENCES}

[1] J. L. Alperin, Local representation theory, Cambridge Studies in Advanced Mathematics, vol. 11, Cambridge University Press, Cambridge, 1986. Modular representations as an introduction to the local representation theory of finite groups. MR860771 (87i:20002)

[2] Frauke M. Bleher, Universal deformation rings and dihedral defect groups, Trans. Amer. Math. Soc. 361 (2009), no. 7, 3661-3705, DOI 10.1090/S0002-9947-09-04543-7. MR2491895 (2010b:20015)

[3] Frauke M. Bleher, Universal deformation rings and generalized quaternion defect groups, Adv. Math. 225 (2010), no. 3, 1499-1522, DOI 10.1016/j.aim.2010.04.004. MR2673738 (2011i:20013)

[4] Frauke M. Bleher and Ted Chinburg, Universal deformation rings and cyclic blocks, Math. Ann. 318 (2000), no. 4, 805-836, DOI 10.1007/s002080000148. MR1802512 (2001m:20013)

[5] Frauke M. Bleher and Ted Chinburg, Universal deformation rings need not be complete intersections (English, with English and French summaries), C. R. Math. Acad. Sci. Paris 342 (2006), no. 4, 229-232, DOI 10.1016/j.crma.2005.12.006. MR.2196003 (2007b:20053)

[6] Frauke M. Bleher and Ted Chinburg, Universal deformation rings need not be complete intersections, Math. Ann. 337 (2007), no. 4, 739-767, DOI 10.1007/s00208-006-0054-2. MR:2285736 (2008g:11093)

[7] Frauke M. Bleher, Ted Chinburg and Bart de Smit, Inverse Problems for deformation rings, Trans. Amer. Math. Soc. 365 (2013), no. 11, 6149-6165. MR3091278

[8] Frauke M. Bleher, Giovanna Llosent, and Jennifer B. Schaefer, Universal deformation rings and dihedral blocks with two simple modules, J. Algebra 345 (2011), 49-71, DOI 10.1016/j.jalgebra.2011.08.010. MR2842053(2012h:20019)

[9] V. M. Bondarenko and Ju. A. Drozd, The representation type of finite groups (Russian), Zap. Naučn. Sem. Leningrad. Otdel. Mat. Inst. Steklov. (LOMI) 71 (1977), 24-41, 282. Modules and representations. MR0472984 (57 \#12663)

[10] Richard Brauer, On the connection between the ordinary and the modular characters of groups of finite order, Ann. of Math. (2) 42 (1941), 926-935. MR0005732 (3,196c)

[11] Richard Brauer, Zur Darstellungstheorie der Gruppen endlicher Ordnung. II (German), Math. Z. 72 (1959/1960), 25-46. MR0108542 (21 \#7258)

[12] Richard Brauer, Some applications of the theory of blocks of characters of finite groups. III, J. Algebra 3 (1966), 225-255. MR0202857 (34 \#2716)

[13] Richard Brauer, On blocks and sections in finite groups. II, Amer. J. Math. 90 (1968), 895925. MR0244398 (39 \#5713)

[14] Richard Brauer, Some applications of the theory of blocks of characters of finite groups. IV, J. Algebra 17 (1971), 489-521. MR0281806 (43 \#7520)

[15] Richard Brauer, On 2-blocks with dihedral defect groups, Symposia Mathematica, Vol. XIII (Convegno di Gruppi e loro Rappresentazioni, INDAM, Rome, 1972), Academic Press, London, 1974, pp. 367-393. MR0354838(50 \#7315)

[16] Sheila Brenner, Modular representations of p groups, J. Algebra 15 (1970), 89-102. MR.0255704 (41 \#364) 
[17] M. C. R. Butler and Claus Michael Ringel, Auslander-Reiten sequences with few middle terms and applications to string algebras, Comm. Algebra 15 (1987), no. 1-2, 145-179, DOI 10.1080/00927878708823416. MR876976 (88a:16055)

[18] Jon F. Carlson and Jacques Thévenaz, The classification of endo-trivial modules, Invent. Math. 158 (2004), no. 2, 389-411, DOI 10.1007/s00222-004-0385-0. MR2096798 (2005e:20013)

[19] Jon F. Carlson and Jacques Thévenaz, The classification of torsion endo-trivial modules, Ann. of Math. (2) 162 (2005), no. 2, 823-883, DOI 10.4007/annals.2005.162.823. MR2183283 (2006f:20012)

[20] Ted Chinburg, Can deformation rings of group representations not be local complete intersections? In: Problems from the Workshop on Automorphisms of Curves. Edited by Gunther Cornelissen and Frans Oort, with contributions by I. Bouw, T. Chinburg, Cornelissen, C. Gasbarri, D. Glass, C. Lehr, M. Matignon, Oort, R. Pries and S. Wewers. Rend. Sem. Mat. Univ. Padova 113 (2005), 129-177.

[21] C. W. Curtis and I. Reiner, Methods of representation theory. Vols. I and II. With applications to finite groups and orders, John Wiley \& Sons, Inc., New York, 1981 and 1987. MR 0892316

[22] E. C. Dade, Blocks with cyclic defect groups, Ann. of Math. (2) 84 (1966), 20-48. MR.0200355 (34 \#251)

[23] Bart de Smit and Hendrik W. Lenstra Jr., Explicit construction of universal deformation rings, Modular forms and Fermat's last theorem (Boston, MA, 1995), Springer, New York, 1997, pp. 313-326. MR 1638482

[24] Florian Eisele, p-adic lifting problems and derived equivalences, J. Algebra 356 (2012), 90114, DOI 10.1016/j.jalgebra.2012.01.015. MR2891124

[25] Karin Erdmann, Blocks whose defect groups are Klein four groups: a correction, J. Algebra 76 (1982), no. 2, 505-518, DOI 10.1016/0021-8693(82)90228-9. MR661869 (83g:20013)

[26] K. Erdmann, Algebras and semidihedral defect groups. I, Proc. London Math. Soc. (3) 57 (1988), no. 1, 109-150, DOI 10.1112/plms/s3-57.1.109. MR940432 (89e:20010)

[27] Karin Erdmann, Blocks of tame representation type and related algebras, Lecture Notes in Mathematics, vol. 1428, Springer-Verlag, Berlin, 1990. MR 1064107 (91c:20016)

[28] Paul Fong, A note on splitting fields of representations of finite groups, Illinois J. Math. 7 (1963), 515-520. MR0153741 (27 \#3702)

[29] D. G. Higman, Indecomposable representations at characteristic p, Duke Math. J. 21 (1954), 377-381. MR0067896 (16,794c)

[30] Thorsten Holm, Derived equivalence classification of algebras of dihedral, semidihedral, and quaternion type, J. Algebra 211 (1999), no. 1, 159-205, DOI 10.1006/jabr.1998.7544. MR.1656577 (2000a:16019)

[31] B. Huppert, Endliche Gruppen. I (German), Die Grundlehren der Mathematischen Wissenschaften, Band 134, Springer-Verlag, Berlin, 1967. MR0224703 (37 \#302)

[32] Henning Krause, Maps between tree and band modules, J. Algebra 137 (1991), no. 1, 186-194, DOI 10.1016/0021-8693(91)90088-P. MR1090218 (92j:16010)

[33] Markus Linckelmann, A derived equivalence for blocks with dihedral defect groups, J. Algebra 164 (1994), no. 1, 244-255, DOI 10.1006/jabr.1994.1061. MR1268334 (94m:20030)

[34] Markus Linckelmann, The source algebras of blocks with a Klein four defect group, J. Algebra 167 (1994), no. 3, 821-854, DOI 10.1006/jabr.1994.1214. MR.1287072(95h:20014)

[35] B. Mazur, Deforming Galois representations, Galois groups over Q (Berkeley, CA, 1987), Math. Sci. Res. Inst. Publ., vol. 16, Springer, New York, 1989, pp. 385-437, DOI 10.1007/9781-4613-9649-9_7. MR1012172 (90k:11057)

[36] Jørn Børling Olsson, On 2-blocks with quaternion and quasidihedral defect groups, J. Algebra 36 (1975), no. 2, 212-241. MR0376841 (51 \#13016)

Department of Mathematics, University of Iowa, Iowa City, Iowa 52242-1419

E-mail address: frauke-bleher@uiowa.edu 